\title{
Accumulation, partitioning, and bioavailability of micronutrients in summer maize as affected by phosphorus supply
}

\author{
Wei Zhang, Dun-Yi Liu, Chao Li, Xin-Ping Chen, Chun-Qin Zou* \\ Key Laboratory of Plant-Soil Interactions, Ministry of Education, Center for Resources, Environment and Food Security, China Agricultural University, \\ Beijing 100193, PR China
}

\section{A R T I C L E I N F O}

\section{Article history:}

Received 20 July 2016

Received in revised form 14 March 2017

Accepted 14 March 2017

\section{Keywords:}

Bioavailability

Micronutrient

Partition

Phosphorus fertilization

\begin{abstract}
A B S T R A C T
Decreased micronutrient concentration in cereal grains caused by excessive application of phosphorus (P) fertilizer may contribute to reduce their nutritional quality. To help correct this problem in maize grain, a 3-year field experiment was conducted to determine how P application rate affects micronutrient partitioning in maize shoots and other plant organs and micronutrient bioavailability in grain. Phosphorus application significantly decreased shoot $\mathrm{zinc}(\mathrm{Zn})$ and copper $(\mathrm{Cu})$ concentrations at all growth stages but had no effects on shoot iron ( $\mathrm{Fe}$ ) and manganese ( $\mathrm{Mn}$ ) concentrations. As the P application rate increased, shoot $\mathrm{Zn}$ and $\mathrm{Cu}$ contents decreased, and shoot Fe and $\mathrm{Mn}$ contents increased. The ratios of pre-anthesis to post-anthesis mineral contents were not affected by $\mathrm{P}$ application rate except $\mathrm{Zn}$. P application increased the percentage of $\mathrm{Zn}$ that was allocated to grain and decreased the percentage that was allocated to other tissues, but had no effects on the allocation of other micronutrients among tissues. The bioavailability of $\mathrm{Zn}, \mathrm{Cu}, \mathrm{Fe}$, and $\mathrm{Mn}$ in grain decreased as $\mathrm{P}$ application rate increased. Overall, taking account of grain yield and nutrients concentration, $\mathrm{P}$ fertilizer rates should range from 12.5 to $25.0 \mathrm{~kg} \mathrm{P}^{-1}$ under the local condition. It can be concluded that not only grain yields, but also nutritional quality, should be considered in assessing optimal $P$ rates in maize.
\end{abstract}

(C) 2017 Elsevier B.V. All rights reserved.

\section{Introduction}

Maize (Zea mays L.) is one of the world's leading cereal grains and is very popular because of its diverse functionality as a food for both humans and animals (Nuss and Tanumihardjo, 2010). Maize is also a dietary staple for more than 200 million people and it provides around $15 \%$ of the protein and $20 \%$ of the calories in the world food diet (Brown et al., 1988). As a result, nutrient-biofortification of maize is attracting increasing attention from researchers (Xue et al., 2014). According to recent publications, the target concentrations of zinc ( $\mathrm{Zn}$ ) and iron (Fe) in biofortified maize are $38 \mathrm{mg} \mathrm{kg}^{-1}$ and $60 \mathrm{mg} \mathrm{kg}^{-1}$, respectively (Bouis and Welch, 2010), but the concentrations of $\mathrm{Zn}$ and $\mathrm{Fe}$ in global maize grain are only about $22.0 \mathrm{mg} \mathrm{kg}^{-1}$ and $21.0 \mathrm{mg} \mathrm{kg}^{-1}$, respectively (Gibson, 2012; Welch, 2002).

In the intensive cultivation of high-yielding crop cultivars, at least $30-50 \%$ of the crop yield is attributable to the application of commercial fertilizers (Stewart et al., 2005). Although phosphorus $(P)$ fertilizer can substantially increase maize grain yield (Banaj

\footnotetext{
* Corresponding author.

E-mail addresses: zcq0206@cau.edu.cn, zcq0206@163.com (C.-Q. Zou).
}

et al., 2006), excessive P fertilizer typically does not result in additional yield increases (Bai et al., 2013) but can lead to environmental pollution (Guo et al., 2010; Le et al., 2010) and deficiencies in Zn, copper (Cu), and Fe of maize (Cakmak, 2002; Stein, 2010). In China, for example, approximately $40 \%$ of the soils are $\mathrm{Zn}$ and Fe deficient, and about 30\% are manganese (Mn) and Cu deficient (Liu, 1991). $P$ application can reduce micronutrient concentrations and especially $\mathrm{Zn}$ and $\mathrm{Cu}$ concentrations in wheat grain (Zhang et al., 2012). It is therefore important to clarify how the micronutrient status in maize grain and stover is affected by $\mathrm{P}$ application in the field.

Many studies have reported how the accumulation of minerals in maize shoots is related to maize yields (Bender et al., 2013; Karlen et al., 1988). For instance, maize that yielded $12.0 \mathrm{Mg}$ of grain ha ${ }^{-1}$ accumulated $49.8 \mathrm{~kg}$ of $\mathrm{P}, 0.1 \mathrm{~kg}$ of $\mathrm{Cu}, 1.4 \mathrm{~kg}$ of Fe, $0.5 \mathrm{~kg}$ of Mn, and $0.5 \mathrm{~kg}$ of Zn ha ${ }^{-1}$ (Bender et al., 2013). If the yield increased to 19.3 $\mathrm{Mg} \mathrm{ha}^{-1}$, the accumulation of $\mathrm{P}, \mathrm{Cu}, \mathrm{Fe}, \mathrm{Mn}$, and $\mathrm{Zn}$ at physiological maturity increased to $69.9,0.14,1.9,0.9$, and $0.8 \mathrm{~kg} \mathrm{ha}^{-1}$, respectively (Karlen et al., 1988). Furthermore, Xue et al. (2014), who evaluated shoot micronutrient accumulation at different growth stages under different yield and nitrogen levels, found that the reciprocal internal efficiencies ( $g$ of micronutrient requirement in plant dry matter per $\mathrm{Mg}$ of grain) of $\mathrm{Fe}, \mathrm{Mn}$, and $\mathrm{Cu}$ were not greatly affected by the yield and N levels. Although researchers have 


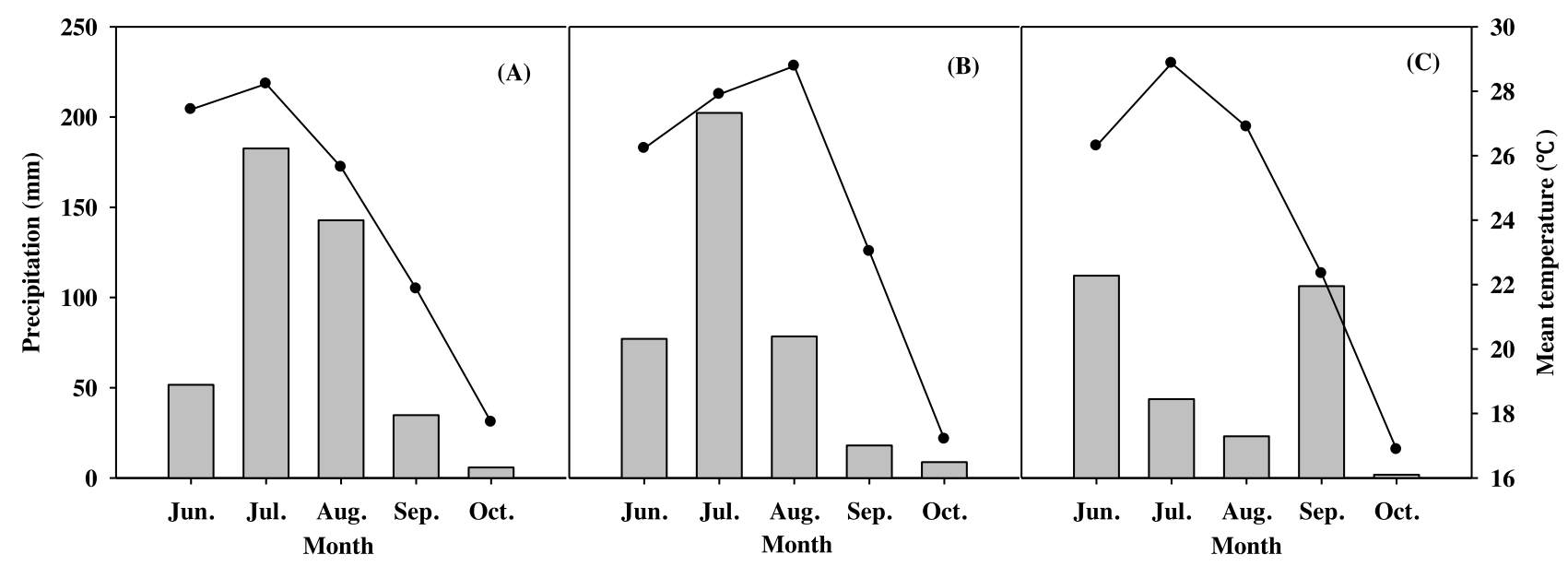

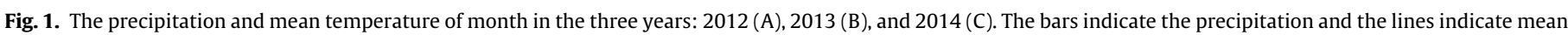
temperature of month.

reported that high rates of $\mathrm{P}$ application can decrease $\mathrm{Zn}$ and $\mathrm{Cu}$ accumulation in wheat (Kizilgoz and Sakin, 2010), little information is available about how the dynamics of micronutrient accumulation (such as $\mathrm{Zn}, \mathrm{Fe}, \mathrm{Mn}$, and $\mathrm{Cu}$ ) in maize are affected by $\mathrm{P}$ application rate. Researchers have also reported that the quantity of minerals accumulated before anthesis determined the quantity that is remobilized into grain of wheat (Stomph et al., 2009). Pearson and Rengel (1994) indicated that a high accumulation of available $\mathrm{Zn}$ pre-anthesis is the main source for the $\mathrm{Zn}$ that is retranslocated to grain. In maize, however, the effect of $P$ application on $Z n$ accumulation pre- and post- anthesis is unclear. Thus, the effect of $P$ fertilizer supply on the accumulation of $\mathrm{Zn}$ and other micronutrients pre- and post- anthesis should be evaluated in maize.

Minerals in plants exhibit different degrees of mobility among tissues, especially during the grain formation stage (Hegelund et al., 2012). While $P$ and $\mathrm{Zn}$ are highly mobile and begin to translocate to maize grain at the $\mathrm{R} 2$ growth stage, $\mathrm{Mn}, \mathrm{Cu}$, and Fe have limited mobility (Bender et al., 2013). A previous study found that large proportions of the $\mathrm{N}$ and $\mathrm{P}$ and a small proportion of $\mathrm{K}$ were translocated from other plant parts to developing grain (Hanway, 1962 ). In another study, about $60 \%$ of the $\mathrm{Zn}, 30 \%$ of the $\mathrm{Cu}, 20 \%$ of the $\mathrm{Fe}$, and $15 \%$ of the Mn originally in maize shoots had accumulated in grain at maturity (Bender et al., 2013). Potential effects of $P$ application levels on $P$ and micronutrient partition among shoot tissues (i.e., among bract, rachis, grain, stem, and leaf) of maize under field conditions has not been studied. In addition, micronutrient bioavailability has been found to be negatively affected by $\mathrm{P}$ application. For instance, Zhang et al. (2012), who used the molar ratio of $\mathrm{P}$ to $\mathrm{Zn}$ to indicate $\mathrm{Zn}$ bioavailability, reported that the increase of $P$ fertilizer rates increased the molar ratio of $P$ to $\mathrm{Zn}$ and therefore decreased $\mathrm{Zn}$ bioavailability in wheat grain.

The aims of this study were to determine how $\mathrm{P}$ application rate affects (1) the temporal dynamics of micronutrient ( $\mathrm{Zn}, \mathrm{Fe}, \mathrm{Mn}$, and $\mathrm{Cu}$ ) accumulation in maize, (2) the partitioning of $\mathrm{P}$ and micronutrients among different maize tissues pre- and post-anthesis, and (3) the micronutrient bioavailability in grain.

\section{Materials and methods}

\subsection{Field location}

A long-term field experiment with maize was conducted at the Quzhou Experiment Station $\left(36.9^{\circ} \mathrm{N}, 115.0^{\circ} \mathrm{E}\right)$ in Hebei Province of China to determine the effect of $P$ fertilizer application on grain yield and nutrients in grain and straw. The experiment was begun in 2008, and the same plots, treatments, and crops were conducted from 2008 to 2014. In this report, all the data of biomass, yield, and nutrients in grain and straw were collected from 2012 to 2014 cropping years. At the beginning of the experiment, the initial soil Olsen-P concentration in 2008 was $7 \mathrm{mg} \mathrm{kg}^{-1}(0.5 \mathrm{M}$ $\mathrm{NaHCO}_{3}$-extractable). The average soil DTPA (Diethylene Triamine Pentacetic Acid)-extractable $\mathrm{Zn}, \mathrm{Cu}, \mathrm{Fe}$, and $\mathrm{Mn}$ concentrations before sowing were $0.40,0.88,8$, and $13 \mathrm{mg} \mathrm{kg}^{-1}$, respectively. The soil micronutrient concentrations were low mainly because the soil was a calcareous alluvial soil with a high $\mathrm{pH}(8.0,1: 2.5 \mathrm{w} / \mathrm{v}$ in water). The soil texture in the location was loam with $140 \mathrm{~g} \mathrm{~kg}^{-1}$ of sand $(2-0.05 \mathrm{~mm}), 605 \mathrm{~g} \mathrm{~kg}^{-1}$ of silt $(0.05-0.002 \mathrm{~mm})$, and $255 \mathrm{~g} \mathrm{~kg}^{-1}$ of clay $(<0.002 \mathrm{~mm})$ on basis of the American soil particle-size classification standard. Meanwhile, the soil $\mathrm{CaCO}_{3}$ and organic matter content were $65 \mathrm{~g} \mathrm{~kg}^{-1}$ and $10.3 \mathrm{~g} \mathrm{~kg}^{-1}$, respectively. The monthly precipitation and mean temperature from a meteorological station installed the Experiment Station are presented in Fig. 1 among the three years.

\subsection{Experimental design}

Six $\mathrm{P}$ application rates, which were applied before sowing in each cropping year, were included in the experiment: $0,12.5,25,50$, 100 , and $200 \mathrm{~kg} \mathrm{P} \mathrm{ha}^{-1}$ (recorded as P0, P12.5, P25, P50, P100, and $\mathrm{P} 200$, respectively). Each treatment was represented by four replicate plots, and each plot was $75 \mathrm{~m}^{2}(7.5 \mathrm{~m} \times 10 \mathrm{~m})$. The plots were arranged in a randomized block design. The summer maize (Zea mays L.) cultivar was 'Nongda 108' in 2012 and 2013 and 'Zhengdan $958^{\prime}$ in 2014 , and the maize density was 67,000 plants ha $^{-1}$. Before sowing, $75 \mathrm{~kg}$ nitrogen (N) ha ${ }^{-1}$ as urea $(46 \% \mathrm{~N}), 60 \mathrm{~kg} \mathrm{~K}_{2} \mathrm{O} \mathrm{ha}^{-1}$ as $\mathrm{K}$ sulphate, and $\mathrm{P}$ fertilizer as calcium superphosphate (7\% P content: $0,179,358,716,1432$, and $2864 \mathrm{~kg} \mathrm{ha}^{-1}$, respectively) were applied. Another $150 \mathrm{~kg} \mathrm{~N} \mathrm{ha}^{-1}$ as urea $(46 \% \mathrm{~N})$ was supplied at the 6-leaf (V6) stage. The $700 \mathrm{~m}^{3} \mathrm{ha}^{-1}$ irrigations were carried out after sowing and at $\mathrm{V} 6$ stage of maize for the three cropping years. Weeds and pests were controlled by standard practices including preemergence herbicide (sprays before emergence of seedlings) and pesticide (sprays at 12-leaf stage) to control weeds and aphids. No water, weed, or pest problems were observed during the experiment. In 2012, 2013, and 2014, the maize seeds were sown on June 12 , June 15 and June 12, respectively and the grain was harvested on October 5, October 4, and October 2, respectively. 


\subsection{Sample collection and nutrient analysis}

Shoot samples of summer maize were collected at the 12leaf (V12) stage, tasseling stage (VT), and physiological maturity stage (R6). At each of these stages, four plants were sampled. To reduce sampling variation, the four maize plants were collected from two adjacent rows with uniform growth height and growth stages which can be the representative of the whole plot. At the R6 stage, the shoot was divided into stem, leaf, bract, rachis, and grain. To evaluate the maize grain yield, all maize ears in a 7.2$\mathrm{m}^{2}(3 \mathrm{~m} \times 2.4 \mathrm{~m})$ area in the center of each plot were harvested. In the study, the grain yield was expressed on a $14.5 \%$ humidity basis. In this report, the term "shoot" refers to all aboveground parts of maize plants including the stover and grain. All samples were rapidly washed with tap water and then with deionized water before they were dried at $60-65^{\circ} \mathrm{C}$ in the oven around $72 \mathrm{~h}$ to constant weight. Then all four plants samples were weighed after the grain was threshed and the harvest index was calculated as grain dry weight divided by total shoot dry weight. All plant samples were ground with a stainless steel grinder for nutrient analysis.

At maturity stage, soil samples were collected to measure the soil Olsen-P concentration and DTPA-Fe, $\mathrm{Mn}, \mathrm{Cu}$, and $\mathrm{Zn}$ concentrations in the three years. In each plot, six subsamples of soil were obtained from a $20 \mathrm{~cm}$ depth using a stainless steel auger then composited into a single sample. To reduce the soil sample variation, the sampling site was following an ' $S$ ' route. Sampling points homogeneously distributed in the plot, while, the soil from field ridge was avoided. All the soils from each plot were air-dried and crushed to pass a $1 \mathrm{~mm}$ sieve prior to analysis. The soil Olsen-P concentration was measured by the molybdovanado phosphate method with $0.5 \mathrm{~mol} \mathrm{~L}^{-1} \mathrm{NaHCO}_{3}$ at $\mathrm{pH}$ 8.5 ( $\left.180 \mathrm{rpm}, 25^{\circ} \mathrm{C}\right)$. The DTPA-Fe, $\mathrm{Mn}, \mathrm{Cu}$, and $\mathrm{Zn}$ concentrations were measured $\left(0.005 \mathrm{~mol} \mathrm{~L}^{-1}\right.$ DTPA-extractable $)$ according to the method described by Lindsay and Norvell (1978). Then nutrients in the extracted solution were measured by inductively coupled plasma optical emission spectroscopy (ICP-OES, OPTIMA 3300 DV, Perkin-Elmer, USA).

The plant samples were digested with $\mathrm{HNO}_{3}-\mathrm{H}_{2} \mathrm{O}_{2}$ in a microwave-accelerated reaction system (CEM, Matthews, NC, USA). The $\mathrm{P}, \mathrm{Zn}, \mathrm{Cu}, \mathrm{Fe}$, and $\mathrm{Mn}$ concentrations in the digested solutions were determined by ICP-OES.

\subsection{Statistical analysis}

Two-way repeated measures analysis of variance (ANOVA) Ftest by SPSS 18.0 was used to assess the effect of $P$ application rate, year, and their interactions on the dependent variables. Before analysis, data normality and variance homogeneity were checked by SPSS $(P>0.05)$. When an ANOVA was significant, treatments were compared by Duncan's multiple comparisons test at the 0.05 probability level $(P<0.05)$. The linear-plateau program of SAS 8.0 was used to quantitatively evaluate the effect of $P$ application on grain yield $(P<0.05)$.

The molar ratios of $\mathrm{P}$ to micronutrients were used to estimate the effect of $P$ application on micronutrient bioavailability. As before, repeated measures ANOVAs were used to assess the effect of $\mathrm{P}$ application rate, year, and interactions on these molar ratios, and means were compared with Duncan's multiple comparisons test. Molar ratios in the grain and stover were calculated as molar mass of $\mathrm{P}$ divided by molar mass of micronutrients.

\section{Results}

\subsection{Grain yield, harvest index (HI), and shoot biomass}

The linear-plateau model showed that 17,22 , and $19 \mathrm{~kg} \mathrm{P} \mathrm{ha}^{-1}$ (estimated values but not real fertilizer rates tested) in 2012, 2013, and 2014 were the threshold fertilizer rates for grain yield. High P application resulted in no increases of grain yield (Fig. 2A). Phosphorus application did not significantly affect the harvest index $(\mathrm{HI})$ (Fig. 2B). Below a $P$ fertilizer rate, shoot biomass at all growth stages increased with increasing $P$ rates $(P<0.05)$ (Fig. 3A-C). For instance, in 2012 shoot biomass significantly increased with $P$ application from 0 to $12.5 \mathrm{~kg} \mathrm{ha}^{-1}$ at V12 and R6 stages then plateaued, whereas the plateau at VT stage appeared at $25 \mathrm{~kg} \mathrm{ha}^{-1} \mathrm{P}$ level. The plateau for shoot biomass also appeared at $25 \mathrm{~kg} \mathrm{ha}^{-1} \mathrm{P}$ rate in 2013 and 2014 at all growth stages, while it was at $50 \mathrm{~kg} \mathrm{ha}^{-1} \mathrm{P}$ rate at VT and R6 stages in 2013.

\subsection{Shoot $P, \mathrm{Zn}, \mathrm{Cu}, \mathrm{Fe}$, and $\mathrm{Mn}$ concentrations and contents at different growth stages as affected by $P$ application rate}

$\mathrm{P}$ application significantly increased the $\mathrm{P}$ concentration in shoots at all three growth stages (Fig. 4A-C). At the V12 stage in 2012, the $P$ concentration in shoots increased with $P$ application from 0 to $25 \mathrm{~kg} \mathrm{ha}^{-1}$ and higher $\mathrm{P}$ rates did not further enhance shoot P concentration; at the V12 stage in 2013 and 2014, the P concentration in shoots increased as the $P$ application rate increased from 0 to $50 \mathrm{~kg} \mathrm{ha}^{-1}$. At the VT stage in the three cropping seasons, the $\mathrm{P}$ concentration in shoots increased as the $\mathrm{P}$ application rate increased from 0 to $50 \mathrm{~kg} \mathrm{ha}^{-1}$. At maturity, the $P$ concentration in shoots continued to increase in response to increasing $P$ application rates (Fig. $4 \mathrm{~A}-\mathrm{C}$ ).

Shoot $\mathrm{Zn}, \mathrm{Cu}, \mathrm{Fe}$, and $\mathrm{Mn}$ concentrations were higher at the V12 stage than at the VT and R6 stages irrespective of P application rate (Fig. 4D-O). Shoot $\mathrm{Zn}$ and $\mathrm{Cu}$ concentrations substantially decreased as the P application rate increased from 0 to $200 \mathrm{~kg} \mathrm{ha}^{-1}$ at all growth stages (Fig. 4D-I). P application had no significant effects on shoot $\mathrm{Fe}$ and $\mathrm{Mn}$ concentrations at all growth stages (Fig. 4J-O). Shoot $\mathrm{P}, \mathrm{Zn}, \mathrm{Cu}, \mathrm{Fe}$, and $\mathrm{Mn}$ contents were greater in the R6 stage than in other stages irrespective of $P$ rate (Fig. 5A-O). The $P$ content at all growth stages increased greatly as the $P$ application rate increased from 0 to $50 \mathrm{~kg} \mathrm{ha}^{-1}$ (the inflection point appeared at $100 \mathrm{~kg} \mathrm{ha}^{-1}$ at maturity in 2014) but was no more increased by higher application rates (Fig. 5A-C). Shoot $\mathrm{Zn}$ and $\mathrm{Cu}$ contents decreased with increasing $P$ rates (Fig. 5D-I), while shoot Fe and Mn contents increased with increasing $P$ rates (Fig. 5J-O).

\subsection{Concentrations and partitioning of $\mathrm{Zn}, \mathrm{Cu}, \mathrm{Fe}$, and $\mathrm{Mn}$ in different tissues of maize at maturity as affected by $P$ application rate}

The concentrations of $\mathrm{Zn}, \mathrm{Cu}, \mathrm{Fe}$, and $\mathrm{Mn}$ in maize tissues varied depending on the $\mathrm{P}$ application rates, years and their interaction (Table 1). Zinc and $\mathrm{Cu}$ concentrations in all tissues significantly decreased with $\mathrm{P}$ application rates. The year also had significantly effect on $\mathrm{Zn}$ and $\mathrm{Cu}$ concentrations in most tissues, while the effect of interaction of $P$ application $\times$ year was not significant. The response of Fe concentration in rachis to $\mathrm{P}$ application varied with year $(P$ value of interaction $=0.000)$. Iron concentrations in stem and leaf increased with $P$ application, meanwhile it was not affected in bracts and grain. The Mn concentrations in all tissues were affected by years, while only in bract and grain were significantly increased by $\mathrm{P}$ application rates (Table 1 ).

The partitioning of $\mathrm{Zn}, \mathrm{Cu}$, and $\mathrm{Mn}$ was affected by $\mathrm{P}$ application rate and year, while the $P$ application $\times$ year interaction had effect on Fe partitioning (Table 2). Phosphorus application significantly 
Table 1

Concentrations of $\mathrm{Zn}, \mathrm{Cu}, \mathrm{Fe}$, and $\mathrm{Mn}$ in tissues of summer maize as affected by $\mathrm{P}$ application rate on calcareous soil.

\begin{tabular}{|c|c|c|c|c|c|c|c|c|c|c|c|c|c|c|c|c|c|c|c|c|c|}
\hline \multirow[t]{3}{*}{ Cropping years } & \multirow[t]{3}{*}{$\mathrm{P}$ rates (kg P/ha) } & \multicolumn{20}{|c|}{ Micronutrient concentration $\left(\mathrm{mg} \mathrm{kg}^{-1}\right)$} \\
\hline & & \multicolumn{5}{|l|}{$\mathrm{Zn}$} & \multicolumn{5}{|l|}{$\mathrm{Cu}$} & \multicolumn{6}{|l|}{$\mathrm{Fe}$} & \multicolumn{4}{|l|}{$\mathrm{Mn}$} \\
\hline & & Stem & Leaf & Bract & Rachis & Grain & Stem & Leaf & Bract & Rachis & Grain & Stem & Leaf & Bract & Rachis & Grain & Stem & Leaf & Bract & Rachis & Grain \\
\hline \multirow{6}{*}{2012} & 0 & $7.4 \mathrm{a}$ & $26.9 \mathrm{a}$ & $5.7 \mathrm{a}$ & $4.8 \mathrm{a}$ & $19.2 \mathrm{a}$ & $6.2 \mathrm{a}$ & $14.6 \mathrm{a}$ & $4.3 \mathrm{a}$ & $1.2 \mathrm{a}$ & $1.8 \mathrm{a}$ & $22.8 \mathrm{~b}$ & $164 \mathrm{~b}$ & $56 \mathrm{a}$ & $17 \mathrm{c}$ & $15.0 \mathrm{ab}$ & $6.7 \mathrm{ab}$ & $75 a$ & $14.1 \mathrm{c}$ & $3.5 \mathrm{a}$ & $4.2 \mathrm{ab}$ \\
\hline & 12.5 & $5.6 \mathrm{ab}$ & $18.8 \mathrm{~b}$ & $3.6 \mathrm{~b}$ & $4.8 \mathrm{a}$ & $16.4 \mathrm{~b}$ & $5.8 \mathrm{ab}$ & $13.5 \mathrm{a}$ & $4.0 \mathrm{ab}$ & $1.0 \mathrm{ab}$ & $1.5 \mathrm{~b}$ & $23.5 \mathrm{~b}$ & $184 \mathrm{ab}$ & 58 a & $17 \mathrm{c}$ & $15.0 \mathrm{ab}$ & $6.4 \mathrm{~b}$ & $67 \mathrm{a}$ & $17.1 \mathrm{a}$ & $3.3 \mathrm{a}$ & $4.0 \mathrm{~b}$ \\
\hline & 25 & $5.2 \mathrm{~b}$ & $15.9 \mathrm{c}$ & $4.3 \mathrm{~b}$ & $3.8 \mathrm{a}$ & $14.2 \mathrm{c}$ & $5.3 \mathrm{~b}$ & $11.5 \mathrm{~b}$ & $3.8 \mathrm{ab}$ & $0.8 \mathrm{bc}$ & $1.5 \mathrm{~b}$ & $23.6 \mathrm{~b}$ & $185 a b$ & $58 \mathrm{a}$ & $19 \mathrm{bc}$ & $14.2 \mathrm{~b}$ & $7.9 \mathrm{a}$ & $72 \mathrm{a}$ & $16.7 \mathrm{ab}$ & $2.9 \mathrm{a}$ & $3.9 \mathrm{~b}$ \\
\hline & 50 & $3.7 \mathrm{bc}$ & $13.8 \mathrm{~cd}$ & $2.4 \mathrm{c}$ & $2.3 \mathrm{~b}$ & 14.8 bc & $4.5 \mathrm{c}$ & $11.6 \mathrm{~b}$ & $3.6 \mathrm{~b}$ & $1.0 \mathrm{ab}$ & $1.5 \mathrm{~b}$ & $25.2 \mathrm{a}$ & $187 \mathrm{ab}$ & $62 \mathrm{a}$ & $21 \mathrm{ab}$ & $15.5 \mathrm{a}$ & $6.0 \mathrm{~b}$ & $75 \mathrm{a}$ & $16.5 \mathrm{ab}$ & $3.1 \mathrm{a}$ & $4.0 \mathrm{~b}$ \\
\hline & 100 & $3.6 \mathrm{bc}$ & $12.5 \mathrm{~d}$ & $1.8 \mathrm{c}$ & $2.0 \mathrm{~b}$ & $13.5 \mathrm{c}$ & $4.5 \mathrm{c}$ & $11.4 \mathrm{~b}$ & $3.5 \mathrm{~b}$ & $0.8 \mathrm{bc}$ & $1.2 \mathrm{c}$ & $25.2 \mathrm{a}$ & $186 \mathrm{ab}$ & $60 \mathrm{a}$ & $20 \mathrm{ab}$ & $14.3 \mathrm{ab}$ & $6.3 \mathrm{~b}$ & $76 \mathrm{a}$ & $17.5 \mathrm{a}$ & $2.9 \mathrm{a}$ & $4.1 \mathrm{ab}$ \\
\hline & 200 & $2.3 \mathrm{c}$ & $13.6 \mathrm{~cd}$ & $1.8 \mathrm{c}$ & $2.0 \mathrm{~b}$ & $13.7 \mathrm{c}$ & $3.5 \mathrm{~d}$ & $11.1 \mathrm{~b}$ & $3.4 \mathrm{~b}$ & $0.5 \mathrm{c}$ & $1.2 \mathrm{c}$ & $24.8 \mathrm{a}$ & $202 \mathrm{a}$ & 63 a & $23 \mathrm{a}$ & $14.5 \mathrm{ab}$ & $6.0 \mathrm{~b}$ & 76 a & $15.3 \mathrm{bc}$ & $3.0 \mathrm{a}$ & $4.4 \mathrm{a}$ \\
\hline \multirow[t]{6}{*}{2013} & 0 & $11.7 \mathrm{a}$ & $29.9 \mathrm{a}$ & $10.2 \mathrm{a}$ & $7.7 \mathrm{a}$ & $22.2 \mathrm{a}$ & $6.6 \mathrm{a}$ & $14.3 \mathrm{a}$ & $5.0 \mathrm{a}$ & $2.4 \mathrm{a}$ & $1.9 \mathrm{a}$ & $33.3 \mathrm{c}$ & $207 \mathrm{~b}$ & $76 a$ & $18 \mathrm{~b}$ & $18.5 \mathrm{ab}$ & $9.5 \mathrm{a}$ & $105 a b$ & $23.5 \mathrm{bc}$ & $3.6 \mathrm{a}$ & $5.8 \mathrm{~b}$ \\
\hline & 12.5 & $7.2 \mathrm{~b}$ & $21.7 \mathrm{~b}$ & $7.8 \mathrm{~b}$ & $7.8 \mathrm{a}$ & $21.0 \mathrm{ab}$ & $5.9 \mathrm{a}$ & $12.4 \mathrm{~b}$ & $4.5 \mathrm{abc}$ & $2.2 \mathrm{a}$ & $1.5 \mathrm{~b}$ & $35.6 \mathrm{c}$ & $226 \mathrm{a}$ & $76 a$ & $25 \mathrm{a}$ & $19.4 \mathrm{a}$ & $8.0 \mathrm{a}$ & $97 \mathrm{~b}$ & $22.5 \mathrm{c}$ & $3.9 \mathrm{a}$ & $6.1 \mathrm{ab}$ \\
\hline & 25 & $6.4 \mathrm{bc}$ & $19.1 \mathrm{c}$ & $6.4 \mathrm{bc}$ & $5.2 \mathrm{~b}$ & $20.3 \mathrm{ab}$ & $5.2 \mathrm{~b}$ & $12.6 \mathrm{~b}$ & $4.6 \mathrm{ab}$ & $2.1 \mathrm{ab}$ & $1.4 \mathrm{bc}$ & $40.8 \mathrm{~b}$ & $236 \mathrm{a}$ & $66 \mathrm{a}$ & 26 a & $17.0 \mathrm{bc}$ & $9.0 \mathrm{a}$ & $103 a b$ & $24.7 \mathrm{abc}$ & $3.9 \mathrm{a}$ & $6.5 \mathrm{a}$ \\
\hline & 50 & $6.0 \mathrm{bcd}$ & $18.3 \mathrm{~cd}$ & $5.9 \mathrm{bc}$ & $3.9 \mathrm{~b}$ & $18.9 \mathrm{bc}$ & $4.8 \mathrm{~b}$ & $12.6 \mathrm{~b}$ & $4.2 \mathrm{bcd}$ & $1.9 \mathrm{~b}$ & $1.2 \mathrm{~cd}$ & $42.7 \mathrm{ab}$ & $232 \mathrm{a}$ & $74 \mathrm{a}$ & $25 \mathrm{a}$ & $15.8 \mathrm{c}$ & $8.5 \mathrm{a}$ & $107 \mathrm{ab}$ & $26.4 \mathrm{a}$ & $4.0 \mathrm{a}$ & $6.2 \mathrm{ab}$ \\
\hline & 100 & $5.0 \mathrm{~cd}$ & $16.7 \mathrm{~d}$ & $5.2 \mathrm{c}$ & $3.7 \mathrm{~b}$ & $17.7 \mathrm{c}$ & $4.0 \mathrm{c}$ & $11.9 \mathrm{~b}$ & $3.9 \mathrm{~cd}$ & $1.6 \mathrm{c}$ & $1.1 \mathrm{~d}$ & $44.5 \mathrm{a}$ & $238 \mathrm{a}$ & $78 \mathrm{a}$ & $26 \mathrm{a}$ & $18.5 \mathrm{ab}$ & $9.5 \mathrm{a}$ & $117 \mathrm{a}$ & $27.4 \mathrm{a}$ & $3.9 \mathrm{a}$ & $6.2 \mathrm{ab}$ \\
\hline & 200 & $4.3 \mathrm{~d}$ & $14.5 \mathrm{e}$ & $4.4 \mathrm{c}$ & $3.1 \mathrm{~b}$ & $17.4 \mathrm{c}$ & $3.7 \mathrm{c}$ & $10.4 \mathrm{c}$ & $3.6 \mathrm{~d}$ & $1.2 \mathrm{~d}$ & $1.0 \mathrm{~d}$ & $41.7 \mathrm{ab}$ & $240 \mathrm{a}$ & $74 \mathrm{a}$ & $25 \mathrm{a}$ & $17.3 \mathrm{ab}$ & $8.4 \mathrm{a}$ & $108 \mathrm{ab}$ & $25.7 \mathrm{ab}$ & $4.1 \mathrm{a}$ & $6.2 \mathrm{ab}$ \\
\hline \multirow[t]{6}{*}{2014} & 0 & $7.9 \mathrm{a}$ & $14.6 \mathrm{a}$ & $7.9 \mathrm{a}$ & $8.0 \mathrm{a}$ & $18.3 \mathrm{a}$ & $5.5 \mathrm{ab}$ & $12.2 \mathrm{a}$ & $4.6 \mathrm{a}$ & $2.5 \mathrm{a}$ & $2.0 \mathrm{a}$ & $32.6 \mathrm{~d}$ & $323 \mathrm{~b}$ & $61 \mathrm{a}$ & $25 \mathrm{~b}$ & $20.2 \mathrm{a}$ & $6.5 \mathrm{a}$ & $88 \mathrm{ab}$ & $18.8 \mathrm{~d}$ & $3.3 \mathrm{~b}$ & $4.1 \mathrm{c}$ \\
\hline & 12.5 & $5.2 \mathrm{~b}$ & $9.8 \mathrm{~b}$ & $5.7 \mathrm{~b}$ & $5.1 \mathrm{~b}$ & $15.7 \mathrm{~b}$ & $5.8 \mathrm{a}$ & $9.4 \mathrm{~b}$ & $4.0 \mathrm{~b}$ & $2.5 \mathrm{a}$ & $1.9 \mathrm{ab}$ & $32.6 \mathrm{~d}$ & 337 a & $64 \mathrm{a}$ & $28 \mathrm{a}$ & $20.5 \mathrm{a}$ & $5.2 \mathrm{ab}$ & $72 c$ & $19.8 \mathrm{~cd}$ & $3.9 \mathrm{a}$ & $4.6 \mathrm{~b}$ \\
\hline & 25 & $4.7 \mathrm{bc}$ & $9.7 \mathrm{~b}$ & $5.4 \mathrm{bc}$ & $4.0 \mathrm{bc}$ & $15.3 \mathrm{~b}$ & $5.4 \mathrm{ab}$ & $9.6 \mathrm{~b}$ & $4.1 \mathrm{~b}$ & $2.3 \mathrm{a}$ & $1.9 \mathrm{ab}$ & $34.6 \mathrm{bcd}$ & 346 a & $60 \mathrm{a}$ & $28 \mathrm{a}$ & $20.5 \mathrm{a}$ & $6.3 \mathrm{ab}$ & $81 \mathrm{bc}$ & $20.7 \mathrm{bc}$ & $3.6 \mathrm{ab}$ & $4.6 \mathrm{~b}$ \\
\hline & 50 & $4.4 \mathrm{bc}$ & $8.8 \mathrm{~b}$ & $4.2 \mathrm{~cd}$ & $3.1 \mathrm{~cd}$ & $13.0 \mathrm{c}$ & $4.5 \mathrm{bc}$ & $8.8 \mathrm{bc}$ & $3.9 \mathrm{~b}$ & $2.4 \mathrm{a}$ & $1.8 \mathrm{bc}$ & $35.5 \mathrm{ab}$ & $343 \mathrm{a}$ & $61 \mathrm{a}$ & $29 a$ & $19.5 \mathrm{a}$ & $6.2 \mathrm{ab}$ & $93 \mathrm{a}$ & $21.4 \mathrm{ab}$ & $3.5 \mathrm{~b}$ & $4.4 \mathrm{bc}$ \\
\hline & 100 & $4.3 \mathrm{bc}$ & $8.1 \mathrm{~b}$ & $3.5 \mathrm{~d}$ & $2.0 \mathrm{~d}$ & $13.1 \mathrm{c}$ & $4.1 \mathrm{c}$ & $7.6 \mathrm{~cd}$ & $3.8 \mathrm{bc}$ & $1.8 \mathrm{~b}$ & $1.7 \mathrm{~cd}$ & $37.7 \mathrm{a}$ & $340 \mathrm{a}$ & $63 \mathrm{a}$ & $29 a$ & $18.8 \mathrm{a}$ & $5.1 \mathrm{~b}$ & $75 c$ & $22.5 \mathrm{a}$ & $3.5 \mathrm{~b}$ & $4.6 \mathrm{~b}$ \\
\hline & 200 & $3.6 \mathrm{c}$ & $7.9 \mathrm{~b}$ & $3.1 \mathrm{~d}$ & $1.9 \mathrm{~d}$ & $12.8 \mathrm{c}$ & $3.8 \mathrm{c}$ & $6.5 \mathrm{~d}$ & $3.5 \mathrm{c}$ & $1.5 \mathrm{~b}$ & $1.5 \mathrm{~d}$ & 34.9 bc & 339 a & $61 \mathrm{a}$ & $27 \mathrm{a}$ & $19.4 \mathrm{a}$ & $5.4 \mathrm{ab}$ & $79 \mathrm{bc}$ & $20.5 b c$ & $3.5 \mathrm{~b}$ & $5.2 \mathrm{a}$ \\
\hline \multicolumn{22}{|c|}{ Source of variation } \\
\hline \multirow{2}{*}{\multicolumn{2}{|c|}{$\begin{array}{l}\text { P application (P) } \\
\text { Year (Y) }\end{array}$}} & 0.000 & 0.000 & 0.000 & 0.000 & 0.000 & 0.000 & 0.000 & 0.000 & 0.000 & 0.000 & 0.000 & 0.002 & 0.136 & 0.000 & 0.133 & 0.056 & 0.054 & 0.000 & 0.545 & 0.001 \\
\hline & & 0.001 & 0.000 & 0.000 & 0.000 & 0.000 & 0.718 & 0.000 & 0.01 & 0.000 & 0.000 & 0.000 & 0.000 & 0.000 & 0.000 & 0.000 & 0.000 & 0.000 & 0.000 & 0.000 & 0.000 \\
\hline \multicolumn{2}{|l|}{$\mathrm{P}^{* \mathrm{Y}}$} & 0.071 & 0.103 & 0.783 & 0.178 & 0.280 & 0.286 & 0.053 & 0.352 & 0.101 & 0.061 & 0.053 & 0.941 & 0.062 & 0.000 & 0.075 & 0.366 & 0.102 & 0.143 & 0.186 & 0.140 \\
\hline
\end{tabular}

Values represent the mean of four replicates. Within a column, different letters denote significant difference at $P<0.05$ by Duncan's multiple comparison test. 
Table 2

Distribution of $\mathrm{Zn}, \mathrm{Cu}, \mathrm{Fe}$, and $\mathrm{Mn}$ in tissues of summer maize as affected by $\mathrm{P}$ application rate on calcareous soil.

\begin{tabular}{|c|c|c|c|c|c|c|c|c|c|c|c|c|c|c|c|c|c|c|c|c|c|}
\hline \multirow[t]{3}{*}{ Cropping years } & \multirow[t]{3}{*}{ P rates (kg P/ha) } & \multicolumn{20}{|c|}{ Distribution of micronutrient (\%) } \\
\hline & & \multicolumn{5}{|l|}{$\mathrm{Zn}$} & \multicolumn{5}{|l|}{$\mathrm{Cu}$} & \multicolumn{5}{|l|}{$\mathrm{Fe}$} & \multicolumn{5}{|l|}{ Mn } \\
\hline & & Stem & Leaf & Bract & Rachis & Grain & Stem & Leaf & Bract & Rachis & Grain & Stem & Leaf & Bract & Rachis & Grain & Stem & Leaf & Bract & Rachis & Grain \\
\hline \multirow[t]{6}{*}{2012} & 0 & $7.3 \mathrm{a}$ & $29.6 \mathrm{a}$ & $2.1 \mathrm{a}$ & $2.2 \mathrm{ab}$ & $58.9 \mathrm{~b}$ & $20.6 \mathrm{a}$ & $54.3 \mathrm{ab}$ & $5.4 \mathrm{ab}$ & $1.8 \mathrm{ab}$ & $17.9 \mathrm{bc}$ & $8.3 \mathrm{ab}$ & $66 \mathrm{ab}$ & $5.4 \mathrm{~b}$ & $2.9 \mathrm{ab}$ & $17.0 \mathrm{a}$ & $6.1 \mathrm{ab}$ & $75.6 \mathrm{a}$ & $4.9 \mathrm{~b}$ & $1.5 \mathrm{a}$ & $12.0 \mathrm{a}$ \\
\hline & 12.5 & $6.5 \mathrm{a}$ & $26.9 \mathrm{ab}$ & $1.7 \mathrm{ab}$ & $2.8 \mathrm{a}$ & $62.2 \mathrm{~b}$ & $19.9 \mathrm{a}$ & $56.7 \mathrm{a}$ & $4.7 \mathrm{~b}$ & $1.7 \mathrm{ab}$ & $16.9 \mathrm{c}$ & $7.3 \mathrm{~b}$ & $70 \mathrm{a}$ & $5.0 \mathrm{~b}$ & $2.7 \mathrm{~b}$ & $15.2 \mathrm{abc}$ & $5.8 \mathrm{ab}$ & $74.7 \mathrm{a}$ & $6.1 \mathrm{a}$ & $1.5 \mathrm{a}$ & $11.9 \mathrm{a}$ \\
\hline & 25 & $6.9 \mathrm{a}$ & $25.5 \mathrm{abc}$ & $2.2 \mathrm{a}$ & $2.5 \mathrm{ab}$ & $62.9 \mathrm{~b}$ & $20.5 \mathrm{a}$ & $53.8 \mathrm{ab}$ & $5.7 \mathrm{a}$ & $1.5 \mathrm{ab}$ & $18.5 \mathrm{abc}$ & $7.4 \mathrm{~b}$ & $68 \mathrm{ab}$ & $6.9 \mathrm{a}$ & $3.0 \mathrm{ab}$ & $14.7 \mathrm{bc}$ & $6.7 \mathrm{a}$ & $75.3 \mathrm{a}$ & $5.5 \mathrm{ab}$ & $1.2 \mathrm{a}$ & $11.2 \mathrm{a}$ \\
\hline & 50 & $5.5 \mathrm{ab}$ & $21.6 \mathrm{c}$ & $1.3 \mathrm{bc}$ & $1.7 \mathrm{bc}$ & $69.9 \mathrm{a}$ & $19.6 \mathrm{a}$ & $51.6 \mathrm{~b}$ & $5.7 \mathrm{a}$ & $2.2 \mathrm{a}$ & $20.8 \mathrm{a}$ & $8.5 \mathrm{ab}$ & $65 \mathrm{~b}$ & $6.8 \mathrm{a}$ & $3.5 \mathrm{a}$ & $16.6 \mathrm{ab}$ & $5.8 \mathrm{ab}$ & $74.7 \mathrm{a}$ & $5.8 \mathrm{a}$ & $1.5 \mathrm{a}$ & $12.2 \mathrm{a}$ \\
\hline & 100 & $6.4 \mathrm{a}$ & $22.6 \mathrm{bc}$ & $1.0 \mathrm{c}$ & $1.5 \mathrm{c}$ & $68.6 \mathrm{a}$ & $20.6 \mathrm{a}$ & $53.0 \mathrm{ab}$ & $6.0 \mathrm{a}$ & $1.6 \mathrm{ab}$ & $18.9 \mathrm{abc}$ & $8.9 \mathrm{a}$ & $67 \mathrm{ab}$ & $6.9 \mathrm{a}$ & $2.9 \mathrm{ab}$ & $14.6 \mathrm{bc}$ & $6.2 \mathrm{ab}$ & $75.4 \mathrm{a}$ & $5.6 \mathrm{ab}$ & $1.2 \mathrm{a}$ & $11.7 \mathrm{a}$ \\
\hline & 200 & $3.7 \mathrm{~b}$ & $24.8 \mathrm{abc}$ & $1.0 \mathrm{c}$ & $1.5 \mathrm{c}$ & $69.0 \mathrm{a}$ & $15.9 \mathrm{a}$ & $57.1 \mathrm{a}$ & $5.6 \mathrm{a}$ & $1.2 \mathrm{~b}$ & $20.3 \mathrm{ab}$ & $7.5 \mathrm{~b}$ & $69 \mathrm{ab}$ & $6.8 \mathrm{a}$ & $3.5 \mathrm{a}$ & $13.6 \mathrm{c}$ & $5.1 \mathrm{~b}$ & $76.3 \mathrm{a}$ & $4.9 \mathrm{~b}$ & $1.3 \mathrm{a}$ & $12.3 \mathrm{a}$ \\
\hline \multirow[t]{6}{*}{2013} & 0 & $9.2 \mathrm{a}$ & $27.7 \mathrm{a}$ & $4.0 \mathrm{a}$ & $3.9 \mathrm{ab}$ & $55.2 \mathrm{~d}$ & $19.7 \mathrm{a}$ & $50.2 \mathrm{a}$ & $7.4 \mathrm{a}$ & $4.5 \mathrm{a}$ & $18.2 \mathrm{a}$ & $8.7 \mathrm{bc}$ & $63 \mathrm{ab}$ & $10.0 \mathrm{a}$ & $3.0 \mathrm{~b}$ & $15.4 \mathrm{ab}$ & $5.8 \mathrm{a}$ & $74.7 \mathrm{a}$ & $7.1 \mathrm{a}$ & $1.4 \mathrm{a}$ & $11.1 \mathrm{~b}$ \\
\hline & 12.5 & $6.0 \mathrm{~b}$ & $22.2 \mathrm{~b}$ & $3.4 \mathrm{ab}$ & $4.4 \mathrm{a}$ & $64.0 \mathrm{c}$ & $19.3 \mathrm{a}$ & $49.8 \mathrm{a}$ & $7.9 \mathrm{a}$ & $4.7 \mathrm{a}$ & $18.4 \mathrm{a}$ & $8.1 \mathrm{c}$ & $63 \mathrm{ab}$ & $9.4 \mathrm{a}$ & $3.6 \mathrm{ab}$ & $16.1 \mathrm{a}$ & $4.8 \mathrm{a}$ & $72.6 \mathrm{ab}$ & $7.4 \mathrm{a}$ & $1.5 \mathrm{a}$ & $13.6 \mathrm{a}$ \\
\hline & 25 & $6.2 \mathrm{~b}$ & $21.0 \mathrm{bc}$ & $2.9 \mathrm{~b}$ & $3.2 \mathrm{ab}$ & $66.7 b c$ & $18.8 \mathrm{ab}$ & $52.0 \mathrm{a}$ & $8.0 \mathrm{a}$ & $4.7 \mathrm{a}$ & $16.6 \mathrm{a}$ & $9.9 \mathrm{ab}$ & $65 \mathrm{a}$ & $7.7 a$ & $3.8 \mathrm{a}$ & $14.0 \mathrm{~b}$ & $5.6 \mathrm{a}$ & $72.1 \mathrm{ab}$ & $7.3 \mathrm{a}$ & $1.5 \mathrm{a}$ & $13.6 \mathrm{a}$ \\
\hline & 50 & $5.8 \mathrm{~b}$ & $20.6 \mathrm{bc}$ & $2.9 \mathrm{~b}$ & $2.4 \mathrm{~b}$ & $68.4 \mathrm{ab}$ & $17.4 \mathrm{ab}$ & $54.2 \mathrm{a}$ & $7.9 \mathrm{a}$ & $4.3 \mathrm{ab}$ & $16.2 \mathrm{a}$ & $10.1 \mathrm{ab}$ & $64 \mathrm{ab}$ & $8.9 a$ & $3.6 \mathrm{ab}$ & $13.9 \mathrm{~b}$ & $5.0 \mathrm{a}$ & $72.3 \mathrm{ab}$ & $7.8 \mathrm{a}$ & $1.5 \mathrm{a}$ & $13.6 \mathrm{a}$ \\
\hline & 100 & $5.3 \mathrm{~b}$ & $19.3 \mathrm{bc}$ & $3.0 \mathrm{~b}$ & $2.7 \mathrm{ab}$ & $69.7 \mathrm{ab}$ & $16.3 \mathrm{~b}$ & $53.6 \mathrm{a}$ & $8.5 \mathrm{a}$ & $4.6 \mathrm{a}$ & $17.0 \mathrm{a}$ & $10.4 \mathrm{a}$ & $60 \mathrm{~b}$ & $9.7 \mathrm{a}$ & $4.1 \mathrm{a}$ & $15.8 \mathrm{ab}$ & $5.4 \mathrm{a}$ & $71.9 \mathrm{ab}$ & $8.3 \mathrm{a}$ & $1.5 \mathrm{a}$ & $13.0 \mathrm{a}$ \\
\hline & 200 & $5.1 \mathrm{~b}$ & $18.1 \mathrm{c}$ & $2.7 \mathrm{~b}$ & $2.3 \mathrm{~b}$ & $71.9 \mathrm{a}$ & $17.6 \mathrm{ab}$ & $52.3 \mathrm{a}$ & $8.8 \mathrm{a}$ & $3.7 \mathrm{~b}$ & $17.7 \mathrm{a}$ & $10.1 \mathrm{ab}$ & $62 \mathrm{ab}$ & $9.3 \mathrm{a}$ & $3.8 \mathrm{a}$ & $14.9 \mathrm{ab}$ & $5.2 \mathrm{a}$ & $71.3 \mathrm{~b}$ & $8.3 \mathrm{a}$ & $1.6 \mathrm{a}$ & $13.8 \mathrm{a}$ \\
\hline \multirow[t]{6}{*}{2014} & 0 & $7.5 \mathrm{~b}$ & $21.8 \mathrm{a}$ & $5.1 \mathrm{a}$ & $4.5 \mathrm{a}$ & $61.1 \mathrm{~b}$ & $19.6 \mathrm{a}$ & $46.9 \mathrm{a}$ & $9.0 \mathrm{a}$ & $4.1 \mathrm{ab}$ & $20.4 \mathrm{a}$ & $8.5 \mathrm{a}$ & $66.7 \mathrm{c}$ & $5.2 \mathrm{a}$ & $3.1 \mathrm{a}$ & $16.5 \mathrm{ab}$ & $5.2 \mathrm{a}$ & $71.4 \mathrm{c}$ & $9.6 \mathrm{a}$ & $1.5 \mathrm{a}$ & $12.3 \mathrm{~b}$ \\
\hline & 12.5 & $10.1 \mathrm{a}$ & $19.8 \mathrm{~b}$ & $3.8 \mathrm{~b}$ & $2.9 \mathrm{~b}$ & $63.4 \mathrm{~b}$ & $20.1 \mathrm{a}$ & $45.1 \mathrm{a}$ & $8.1 \mathrm{~b}$ & $4.3 \mathrm{a}$ & $22.4 \mathrm{a}$ & $8.7 \mathrm{a}$ & $66.2 \mathrm{~b}$ & $5.4 \mathrm{a}$ & $2.4 \mathrm{~b}$ & $17.3 \mathrm{a}$ & $4.2 \mathrm{ab}$ & $72.1 \mathrm{c}$ & $9.2 \mathrm{a}$ & $1.6 \mathrm{a}$ & $12.9 \mathrm{ab}$ \\
\hline & 25 & $5.9 \mathrm{~b}$ & $18.9 \mathrm{~b}$ & $3.6 \mathrm{bc}$ & $2.4 \mathrm{~b}$ & $69.2 \mathrm{a}$ & $19.8 \mathrm{a}$ & $47.7 \mathrm{a}$ & $7.9 \mathrm{~b}$ & $4.0 \mathrm{ab}$ & $20.6 \mathrm{a}$ & $8.1 \mathrm{a}$ & $67.7 \mathrm{ab}$ & $5.5 \mathrm{a}$ & $2.3 \mathrm{bc}$ & $16.4 \mathrm{ab}$ & $4.7 \mathrm{ab}$ & $72.9 \mathrm{bc}$ & $8.1 \mathrm{ab}$ & $1.3 \mathrm{~b}$ & $13.0 \mathrm{ab}$ \\
\hline & 50 & $7.2 \mathrm{~b}$ & $18.4 \mathrm{~b}$ & $3.3 \mathrm{bcd}$ & $2.2 \mathrm{~b}$ & $68.9 \mathrm{a}$ & $19.3 \mathrm{a}$ & $47.7 \mathrm{a}$ & $8.2 \mathrm{~b}$ & $4.5 \mathrm{a}$ & $20.3 \mathrm{a}$ & $8.7 \mathrm{a}$ & $68.1 \mathrm{ab}$ & $5.5 \mathrm{a}$ & $2.5 \mathrm{~b}$ & $15.2 \mathrm{~b}$ & $4.5 \mathrm{ab}$ & $74.4 \mathrm{a}$ & $7.4 \mathrm{~b}$ & $1.1 \mathrm{~b}$ & $12.6 \mathrm{ab}$ \\
\hline & 100 & $6.4 \mathrm{~b}$ & $16.6 \mathrm{c}$ & $2.6 \mathrm{~cd}$ & $1.4 \mathrm{c}$ & $73.0 \mathrm{a}$ & $17.8 \mathrm{a}$ & $47.6 \mathrm{a}$ & $8.3 \mathrm{~b}$ & $3.7 \mathrm{~b}$ & $22.6 \mathrm{a}$ & $8.3 \mathrm{a}$ & $69.1 \mathrm{a}$ & $5.1 \mathrm{a}$ & $2.3 \mathrm{bc}$ & $15.2 \mathrm{~b}$ & $3.8 \mathrm{~b}$ & $73.2 \mathrm{~b}$ & $8.4 \mathrm{ab}$ & $1.3 \mathrm{~b}$ & $13.3 \mathrm{ab}$ \\
\hline & 200 & $5.7 \mathrm{~b}$ & $17.9 \mathrm{bc}$ & $2.4 \mathrm{~d}$ & $1.4 \mathrm{c}$ & $72.6 \mathrm{a}$ & $19.9 \mathrm{a}$ & $47.5 \mathrm{a}$ & $8.0 \mathrm{~b}$ & $4.3 \mathrm{a}$ & $20.3 \mathrm{a}$ & $8.2 \mathrm{a}$ & $68.2 \mathrm{ab}$ & $5.5 \mathrm{a}$ & $2.0 \mathrm{c}$ & $16.1 \mathrm{ab}$ & $4.1 \mathrm{ab}$ & $73.2 \mathrm{~b}$ & $7.7 \mathrm{~b}$ & $1.4 \mathrm{~b}$ & $13.6 \mathrm{a}$ \\
\hline \multicolumn{22}{|c|}{ Source of variation } \\
\hline \multicolumn{2}{|c|}{ P application $(\mathrm{P})$} & 0.000 & 0.004 & 0.000 & 0.000 & 0.000 & 0.074 & 0.642 & 0.357 & 0.054 & 0.886 & 0.051 & 0.055 & 0.637 & 0.263 & 0.072 & 0.090 & 0.431 & 0.096 & 0.156 & 0.075 \\
\hline \multirow{2}{*}{\multicolumn{2}{|c|}{$\begin{array}{l}\text { Year }(Y) \\
P^{*} Y\end{array}$}} & 0.580 & 0.000 & 0.000 & 0.001 & 0.000 & 0.245 & 0.000 & 0.000 & 0.000 & 0.000 & 0.000 & 0.000 & 0.000 & 0.000 & 0.000 & 0.001 & 0.001 & 0.000 & 0.005 & 0.031 \\
\hline & & 0.055 & 0.061 & 0.054 & 0.111 & 0.065 & 0.057 & 0.260 & 0.058 & 0.266 & 0.110 & 0.000 & 0.007 & 0.000 & 0.302 & 0.054 & 0.827 & 0.079 & 0.098 & 0.128 & 0.056 \\
\hline
\end{tabular}

Values represent the mean of four replicates. Within a column, different letters denote significant difference at $P<0.05$ by Duncan's multiple comparison test. 

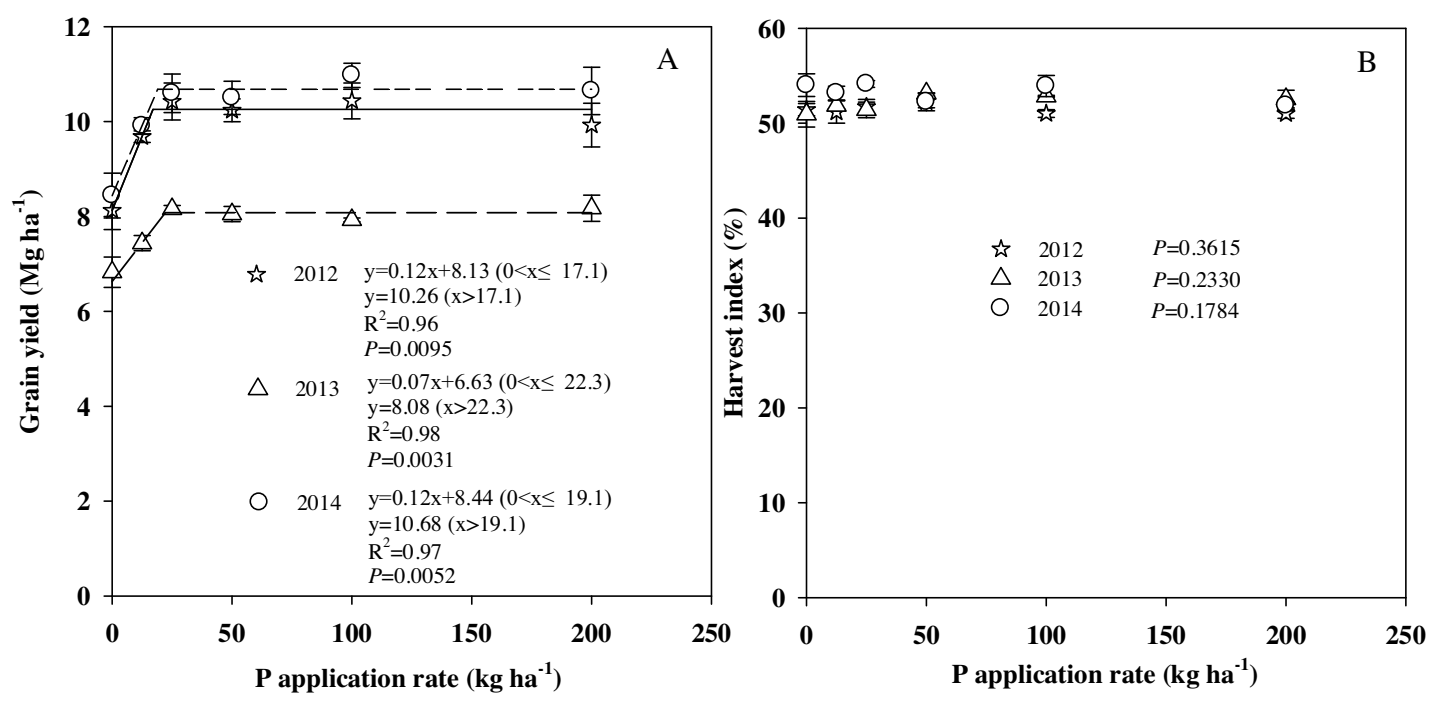

Fig. 2. Grain yield (A) and harvest index (B) of summer maize as affected by P application rate in the three year. Values are means \pm standard error ( $n=4$ ).

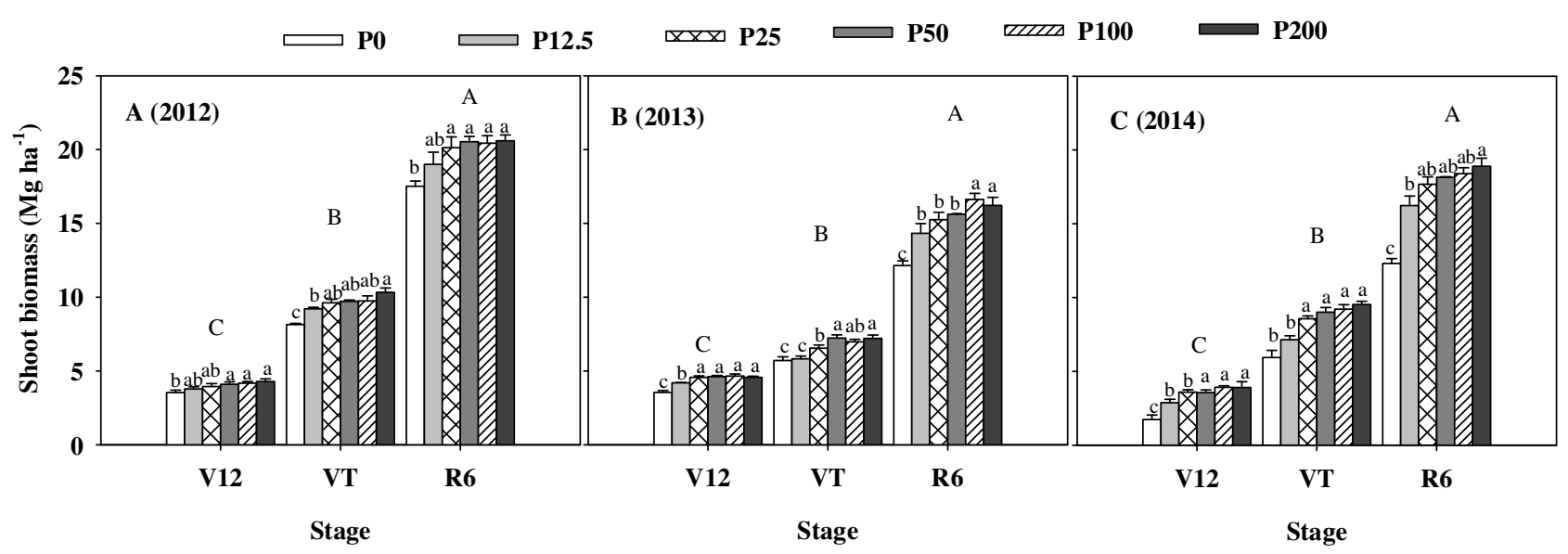

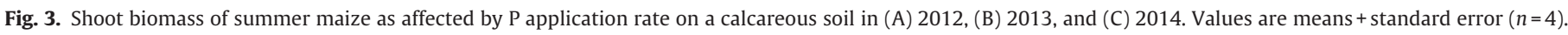

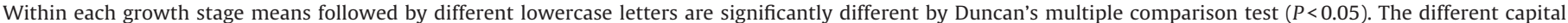
letters indicate a significant difference between growth stage $(P<0.05)$.

increased $\mathrm{Zn}$ distribution in grain and decreased the distribution in other shoot tissues and the year also had a significant effect on Zn distribution. Phosphorus application had no effect on Cu distribution while the year had effect on Cu concentration in leaf, bract, rachis, and grain. The response of Fe concentration in stem, leaf, and bract to increasing $P$ rates varied with year $(P$ value of interaction $<0.05$ ). Phosphorus application had no effect on Mn distribution while the year significantly affected Mn distribution.

\subsection{Molar ratios of $P$ to micronutrients in grain and stover}

The molar ratios of $\mathrm{P} / \mathrm{Zn}, \mathrm{P} / \mathrm{Cu}, \mathrm{P} / \mathrm{Fe}$, and $\mathrm{P} / \mathrm{Mn}$ in grain and stover of mature maize were significantly affected by $\mathrm{P}$ application rate and cropping year. Overall, grain and stover molar ratios of $\mathrm{P} / \mathrm{Zn}$, $\mathrm{P} / \mathrm{Cu}, \mathrm{P} / \mathrm{Fe}$, and $\mathrm{P} / \mathrm{Mn}$ increased as the $\mathrm{P}$ application rate increased from 0 to $200 \mathrm{~kg} \mathrm{ha}^{-1}$ (Table 3 ).

\subsection{Accumulation of $\mathrm{P}, \mathrm{Zn}, \mathrm{Cu}, \mathrm{Fe}$, and $\mathrm{Mn}$ pre- and post-anthesis}

The percentages of the total quantity of minerals accumulated in maize differed pre- vs. post-anthesis (Table 4). Pre-anthesis accumulation accounted for $>80 \%$ of the total $\mathrm{Cu}$ and $\mathrm{Mn}, 50-70 \%$ of the total $\mathrm{P}, \mathrm{Zn}$, and Fe. Phosphorus application increased the ratio of
Zn accumulation but slightly decreased Fe accumulation ratio preanthesis. The ratio of $\mathrm{Mn}$ and $\mathrm{Cu}$ accumulations of pre-anthesis vs. post-anthesis was not affected by $\mathrm{P}$ application rates (Table 4).

\subsection{Effect of $P$ application on soil Olsen-P concentration and DTPA-Fe, $-\mathrm{Mn},-\mathrm{Cu}$, and $-\mathrm{Zn}$ concentration}

Phosphorus application significantly increased soil Olsen-P concentration $(P=0.0012)$. The soil DTPA-Fe, $-\mathrm{Mn}$, and $-\mathrm{Cu}$ concentrations was not affected by $\mathrm{P}$ application rates while soil DTPA-Zn concentration was increased with $\mathrm{P}$ application from 0 to $200 \mathrm{~kg} \mathrm{ha}^{-1}$ (Fig. 6).

\section{Discussion}

\subsection{Shoot biomass and yield}

In agreement with previous report (Banaj et al., 2006; Kovačević et al., 2008), maize grain yield and shoot biomass were significantly increased with P application. It was reported that the critical soil available $P$ concentration to reach the yield plateau was varied in different areas due to the maize cultivars, soil type, $\mathrm{pH}$ and soil organic matter content (Bai et al., 2013). In view of absolute value 

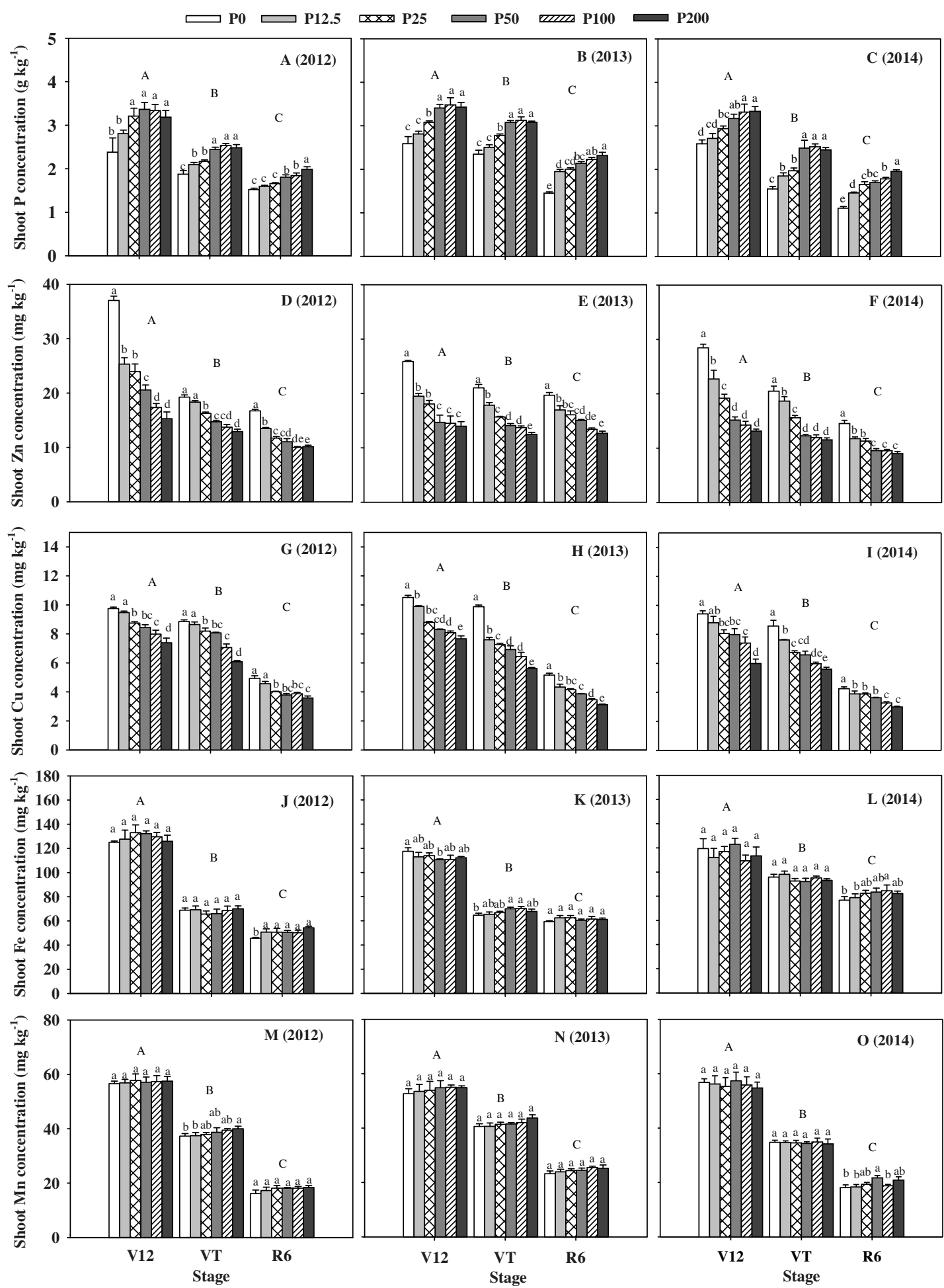

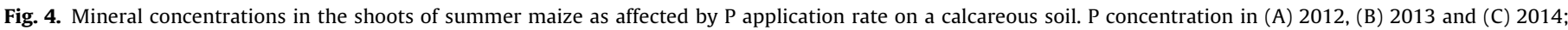

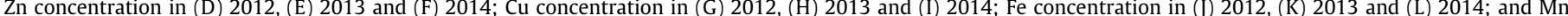

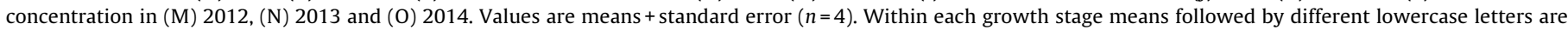
significantly different by Duncan's multiple comparison test $(P<0.05)$. The different capital letters indicate a significant difference between growth stage $(P<0.05)$.

of shoot biomass accumulation, P application also had significant effect on biomass accumulation at early growth stage, indicating that a sufficient P supply at the early growth stage was equally important for maize growth. The grain yield and shoot biomass in 2013 was lower than that in 2012 and 2014, which could be partly explained by differences in climate (precipitation and mean temperature) among the three years. In July of 2013, the higher precipitation accompanied with lower temperature may partly attributed to the decrease in shoot biomass among the stage from V12 to VT stage. 

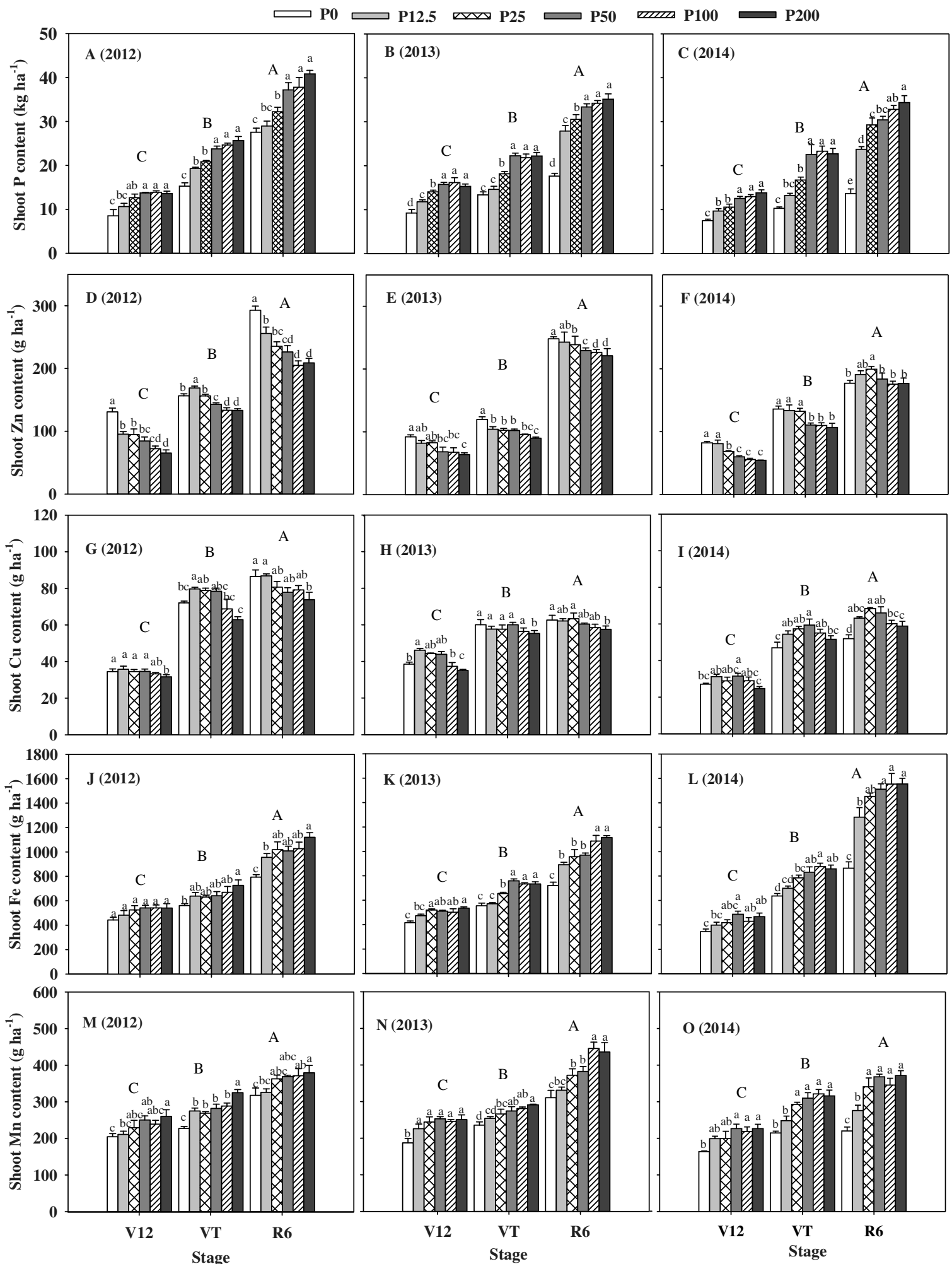

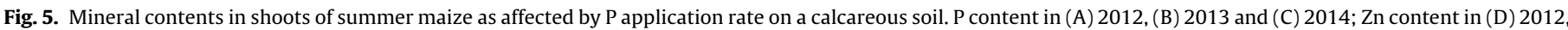

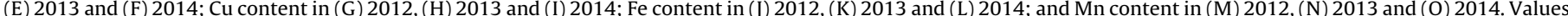

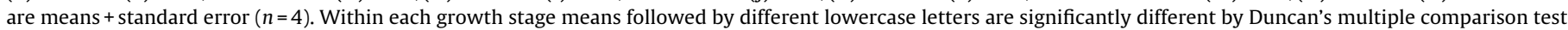
$(P<0.05)$. The different capital letters indicate a significant difference between growth stage $(P<0.05)$.

\subsection{Shoot micronutrient concentration}

As the maize plants grew, the concentrations of micronutrients in the shoots generally decreased but their contents increased. At each growth stage, however, the effects of $\mathrm{P}$ application rate on the concentration and content of each mineral differed. As P application rate increased, the shoot $\mathrm{P}$ concentration substantially increased while the shoot $\mathrm{Zn}$ concentration significantly decreased. In previous studies of wheat, maize, soybean, and cotton, $\mathrm{P}$ application increased the concentration of $\mathrm{P}$ (Colomb et al., 2007) but decreased 
Table 3

The effect of $\mathrm{P}$ application rate on the molar ratios of $\mathrm{P} / \mathrm{Zn}, \mathrm{P} / \mathrm{Cu}, \mathrm{P} / \mathrm{Fe}$, and $\mathrm{P} / \mathrm{Mn}$ in grain and stover of field maize at maturity.

\begin{tabular}{|c|c|c|c|c|c|c|c|c|c|}
\hline \multirow[t]{3}{*}{ Cropping years } & \multirow[t]{3}{*}{$\mathrm{P}$ rates (kg P/ha) } & \multicolumn{8}{|c|}{ Molar ratio of phosphorus to micronutrient } \\
\hline & & \multicolumn{4}{|l|}{ Grain } & \multicolumn{4}{|l|}{ Straw } \\
\hline & & $\mathrm{P} / \mathrm{Zn}$ & $\mathrm{P} / \mathrm{Cu}$ & $\mathrm{P} / \mathrm{Fe}$ & $\mathrm{P} / \mathrm{Mn}$ & $\mathrm{P} / \mathrm{Zn}$ & $\mathrm{P} / \mathrm{Cu}$ & $\mathrm{P} / \mathrm{Fe}$ & $\mathrm{P} / \mathrm{Mn}$ \\
\hline \multirow[t]{6}{*}{2012} & 0 & 273 e & $2990 \mathrm{~d}$ & 298 bc & $1050 \mathrm{~b}$ & $92 \mathrm{f}$ & $153 \mathrm{~d}$ & $14 \mathrm{~cd}$ & $34 \mathrm{c}$ \\
\hline & 12.5 & 303 de & $3240 \mathrm{~cd}$ & $287 \mathrm{c}$ & $1058 \mathrm{~b}$ & $132 \mathrm{e}$ & $173 \mathrm{~d}$ & $13 \mathrm{~d}$ & $37 \mathrm{bc}$ \\
\hline & 25 & $357 \mathrm{~cd}$ & $3498 \mathrm{~cd}$ & 308 bc & $1109 \mathrm{ab}$ & $169 \mathrm{~d}$ & $220 \mathrm{c}$ & $15 \mathrm{bcd}$ & $39 \mathrm{bc}$ \\
\hline & 50 & $397 \mathrm{bc}$ & 3792 bc & 322 b & $1222 \mathrm{a}$ & $233 c$ & 253 bc & $16 \mathrm{abc}$ & $43 \mathrm{~b}$ \\
\hline & 100 & $442 \mathrm{ab}$ & $4128 \mathrm{ab}$ & $358 \mathrm{a}$ & $1221 \mathrm{a}$ & $265 b$ & $262 \mathrm{~b}$ & $17 \mathrm{ab}$ & $44 \mathrm{~b}$ \\
\hline & 200 & $456 \mathrm{a}$ & $4362 \mathrm{a}$ & $372 \mathrm{a}$ & 1199 a & 308 a & $335 a$ & $18 a$ & $51 \mathrm{a}$ \\
\hline \multirow{6}{*}{2013} & 0 & $222 \mathrm{~d}$ & 2559 e & $230 \mathrm{~d}$ & $719 \mathrm{c}$ & 75 e & $153 \mathrm{e}$ & $11 \mathrm{~d}$ & $25 \mathrm{~d}$ \\
\hline & 12.5 & 307 c & $4105 \mathrm{~d}$ & $283 c$ & $888 \mathrm{ab}$ & $129 \mathrm{~d}$ & $216 \mathrm{~d}$ & $13 \mathrm{~cd}$ & $33 c$ \\
\hline & 25 & 319 bc & $4732 \mathrm{c}$ & 328 abc & 851 b & $174 \mathrm{c}$ & $256 c$ & $14 \mathrm{c}$ & $36 \mathrm{bc}$ \\
\hline & 50 & $360 \mathrm{ab}$ & 5695 b & $373 a$ & $923 \mathrm{ab}$ & $186 \mathrm{c}$ & 262 c & $14 \mathrm{c}$ & $35 \mathrm{bc}$ \\
\hline & 100 & 391 a & $6156 a b$ & 325 bc & $945 \mathrm{a}$ & $251 \mathrm{~b}$ & $348 \mathrm{~b}$ & $17 \mathrm{~b}$ & $39 \mathrm{~b}$ \\
\hline & 200 & $399 a$ & 6479 a & $343 a b$ & 939 a & 349 a & $474 \mathrm{a}$ & $20 a$ & $51 \mathrm{a}$ \\
\hline \multirow[t]{6}{*}{2014} & 0 & $185 \mathrm{c}$ & $1657 \mathrm{~d}$ & $144 \mathrm{~d}$ & $908 \mathrm{c}$ & $106 \mathrm{c}$ & $206 \mathrm{~d}$ & $6 c$ & 39 b \\
\hline & 12.5 & $286 \mathrm{~b}$ & 2276 c & $192 \mathrm{c}$ & $1071 \mathrm{~b}$ & $217 \mathrm{~b}$ & $267 c$ & $7 c$ & $47 \mathrm{~b}$ \\
\hline & 25 & $335 \mathrm{~b}$ & $2740 \mathrm{bc}$ & 207 c & $1221 \mathrm{a}$ & $232 \mathrm{~b}$ & $247 \mathrm{~cd}$ & $9 \mathrm{abc}$ & $46 \mathrm{~b}$ \\
\hline & 50 & $409 \mathrm{a}$ & 2953 b & $264 \mathrm{a}$ & $1322 \mathrm{a}$ & $259 \mathrm{~b}$ & $247 \mathrm{~cd}$ & 8 bc & $40 \mathrm{~b}$ \\
\hline & 100 & $402 \mathrm{a}$ & $3110 a b$ & $274 \mathrm{a}$ & $1264 \mathrm{a}$ & $361 \mathrm{a}$ & 359 b & $10 a b$ & $58 \mathrm{a}$ \\
\hline & 200 & $456 a$ & 3702 a & $242 a b$ & $1245 \mathrm{a}$ & $413 \mathrm{a}$ & $422 \mathrm{a}$ & $11 \mathrm{a}$ & $65 a$ \\
\hline \multicolumn{10}{|c|}{ Source of variation } \\
\hline \multicolumn{2}{|c|}{$\mathrm{P}$ application $(\mathrm{P})$} & 0.000 & 0.000 & 0.000 & 0.000 & 0.000 & 0.000 & 0.000 & 0.000 \\
\hline \multicolumn{2}{|l|}{ Year $(\mathrm{Y})$} & 0.030 & 0.000 & 0.000 & 0.000 & 0.000 & 0.000 & 0.000 & 0.000 \\
\hline \multicolumn{2}{|l|}{$\mathrm{P}^{*} \mathrm{Y}$} & 0.162 & 0.090 & 0.145 & 0.052 & 0.061 & 0.102 & 0.178 & 0.128 \\
\hline
\end{tabular}

Values represent the mean of four replicates. Within a column, different letters denote significant difference at $P<0.05$ by Duncan's multiple comparison test.

Table 4

Ratio of content at pre-anthesis vs. post-anthesis to total shoot content of $\mathrm{P}, \mathrm{Zn}, \mathrm{Cu}, \mathrm{Fe}$, and Mn of summer maize on a calcareous soil as affected by $\mathrm{P}$ application rate.

\begin{tabular}{|c|c|c|c|c|c|c|c|c|c|c|c|}
\hline \multirow[t]{3}{*}{ Cropping years } & \multirow[t]{3}{*}{$\mathrm{P}$ rates (kg P/ha) } & \multicolumn{10}{|c|}{ Ratio of minerals content at pre-anthesis and post-anthesis (\%) } \\
\hline & & \multicolumn{2}{|l|}{$\mathrm{P}$} & \multicolumn{2}{|l|}{$\mathrm{Zn}$} & \multicolumn{2}{|l|}{$\mathrm{Cu}$} & \multicolumn{2}{|l|}{$\mathrm{Fe}$} & \multicolumn{2}{|l|}{$\mathrm{Mn}$} \\
\hline & & Pre & Post & Pre & Post & Pre & Post & Pre & Post & Pre & Post \\
\hline \multirow[t]{6}{*}{2012} & 0 & $56 \mathrm{~b}$ & $44 \mathrm{a}$ & $49 \mathrm{~b}$ & $51 \mathrm{a}$ & 95 a & $5 \mathrm{a}$ & $71 \mathrm{a}$ & $29 \mathrm{a}$ & $88 \mathrm{a}$ & $12 \mathrm{a}$ \\
\hline & 12.5 & $67 \mathrm{a}$ & $33 \mathrm{~b}$ & $62 \mathrm{a}$ & $38 \mathrm{~b}$ & $97 \mathrm{a}$ & $3 a$ & $70 \mathrm{a}$ & $30 a$ & $83 a$ & $17 \mathrm{a}$ \\
\hline & 25 & $65 \mathrm{a}$ & $35 \mathrm{~b}$ & $62 \mathrm{a}$ & $38 \mathrm{~b}$ & 99 a & $1 \mathrm{a}$ & $68 \mathrm{a}$ & $32 \mathrm{a}$ & $83 a$ & $17 \mathrm{a}$ \\
\hline & 50 & $64 \mathrm{a}$ & $36 \mathrm{~b}$ & $63 a$ & $37 \mathrm{~b}$ & $96 a$ & $4 \mathrm{a}$ & $64 \mathrm{a}$ & $36 a$ & $82 \mathrm{a}$ & $18 \mathrm{a}$ \\
\hline & 100 & $66 \mathrm{a}$ & $34 \mathrm{~b}$ & $65 a$ & $35 \mathrm{~b}$ & $96 a$ & $4 \mathrm{a}$ & $66 a$ & $34 \mathrm{a}$ & $84 \mathrm{a}$ & $16 a$ \\
\hline & 200 & $63 a$ & $37 \mathrm{~b}$ & $69 a$ & $31 \mathrm{~b}$ & $97 \mathrm{a}$ & $3 \mathrm{a}$ & $65 a$ & $35 \mathrm{a}$ & $83 a$ & $17 \mathrm{a}$ \\
\hline \multirow[t]{6}{*}{2013} & 0 & $66 \mathrm{a}$ & $34 \mathrm{a}$ & $35 \mathrm{~b}$ & $65 a$ & $92 \mathrm{a}$ & $8 \mathrm{a}$ & $51 \mathrm{a}$ & $49 \mathrm{~b}$ & $77 \mathrm{a}$ & $23 a$ \\
\hline & 12.5 & $58 \mathrm{a}$ & $42 \mathrm{a}$ & $44 \mathrm{ab}$ & $56 \mathrm{ab}$ & $93 \mathrm{a}$ & $7 \mathrm{a}$ & $48 \mathrm{ab}$ & $52 \mathrm{ab}$ & $77 \mathrm{a}$ & $23 a$ \\
\hline & 25 & $60 \mathrm{a}$ & $40 \mathrm{a}$ & $44 \mathrm{ab}$ & $56 \mathrm{ab}$ & $88 \mathrm{a}$ & $12 \mathrm{a}$ & $46 \mathrm{ab}$ & $54 \mathrm{ab}$ & $72 \mathrm{a}$ & $28 \mathrm{a}$ \\
\hline & 50 & $67 \mathrm{a}$ & $33 a$ & $45 \mathrm{ab}$ & $55 \mathrm{~b}$ & $99 a$ & $1 \mathrm{a}$ & $46 \mathrm{ab}$ & $54 \mathrm{ab}$ & $72 \mathrm{a}$ & $28 \mathrm{a}$ \\
\hline & 100 & $58 \mathrm{a}$ & $42 \mathrm{a}$ & $46 \mathrm{a}$ & $54 \mathrm{~b}$ & 93 a & $7 \mathrm{a}$ & $45 \mathrm{ab}$ & $55 \mathrm{ab}$ & $63 a$ & $37 \mathrm{a}$ \\
\hline & 200 & $58 \mathrm{a}$ & $42 \mathrm{a}$ & $47 \mathrm{a}$ & $53 \mathrm{~b}$ & $96 \mathrm{a}$ & $4 \mathrm{a}$ & $41 \mathrm{a}$ & 59 a & $68 \mathrm{a}$ & $32 \mathrm{a}$ \\
\hline \multirow[t]{6}{*}{2014} & 0 & $67 \mathrm{a}$ & $33 \mathrm{ab}$ & $52 \mathrm{~b}$ & $48 \mathrm{a}$ & $90 \mathrm{a}$ & $10 \mathrm{a}$ & $74 \mathrm{a}$ & $26 \mathrm{~b}$ & $88 \mathrm{a}$ & $12 \mathrm{a}$ \\
\hline & 12.5 & $63 a$ & $37 \mathrm{ab}$ & $65 a$ & $35 \mathrm{~b}$ & $86 a$ & $14 \mathrm{a}$ & $55 \mathrm{~b}$ & $45 \mathrm{a}$ & $89 a$ & $11 \mathrm{a}$ \\
\hline & 25 & $62 \mathrm{a}$ & $38 \mathrm{a}$ & $67 \mathrm{a}$ & $33 \mathrm{~b}$ & $84 a$ & $16 \mathrm{a}$ & $54 \mathrm{~b}$ & $46 a$ & $87 \mathrm{a}$ & $13 \mathrm{a}$ \\
\hline & 50 & $67 \mathrm{a}$ & $33 \mathrm{ab}$ & $64 \mathrm{a}$ & $36 b$ & $65 \mathrm{a}$ & $35 \mathrm{a}$ & $53 \mathrm{~b}$ & $47 \mathrm{a}$ & $79 \mathrm{a}$ & $21 \mathrm{a}$ \\
\hline & 100 & $71 \mathrm{a}$ & $29 \mathrm{~b}$ & $68 \mathrm{a}$ & $32 \mathrm{~b}$ & $92 \mathrm{a}$ & $8 \mathrm{a}$ & $52 \mathrm{~b}$ & $48 \mathrm{a}$ & $82 \mathrm{a}$ & $18 \mathrm{a}$ \\
\hline & 200 & $62 \mathrm{a}$ & $38 \mathrm{a}$ & $73 a$ & $27 \mathrm{~b}$ & $88 \mathrm{a}$ & $12 \mathrm{a}$ & $49 \mathrm{~b}$ & $51 \mathrm{a}$ & $77 \mathrm{a}$ & $23 a$ \\
\hline \multicolumn{12}{|c|}{ Source of variation } \\
\hline \multicolumn{2}{|l|}{ P application (P) } & 0.275 & 0.275 & 0.000 & 0.000 & 0.641 & 0.641 & 0.000 & 0.000 & 0.174 & 0.174 \\
\hline \multicolumn{2}{|l|}{ Year $(\mathrm{Y})$} & 0.066 & 0.066 & 0.000 & 0.000 & 0.007 & 0.007 & 0.000 & 0.000 & 0.006 & 0.006 \\
\hline \multicolumn{2}{|l|}{$\mathrm{P}^{*} \mathrm{Y}$} & 0.240 & 0.240 & 0.962 & 0.962 & 0.210 & 0.210 & 0.250 & 0.250 & 0.919 & 0.919 \\
\hline
\end{tabular}

Values represent the mean of four replicates. Within a column, different letters denote significant difference at $P<0.05$ by Duncan's multiple comparison test.

the concentration of Zn in stover and grain (Izsáki, 2014; Zhang et al., 2012). At all growth stages in the current study, the Cu concentration in shoots had a similar response as $\mathrm{Zn}$ to $\mathrm{P}$ application rate. Similar results were reported by Zhang et al. (2012) in winter wheat and by Haldar and Mandal (1981) in rice for both shoots and roots. Researchers have often discussed possible explanations for decreased $\mathrm{Zn}$ and $\mathrm{Cu}$ concentrations with increased $\mathrm{P}$ rates. Shukla and Singh (1979) suggested that $P$ application reduced $C u$ availability in soil because of the formation of $\mathrm{Cu}_{3}\left(\mathrm{PO}_{4}\right)_{2}$, which is insoluble.
In the current study, soil DTPA-Zn and DTPA-Cu were not decreased by $\mathrm{P}$ application (Fig. 6B), indicating that the current $\mathrm{P}$ supply did not affect $\mathrm{Zn}$ and $\mathrm{Cu}$ solubility in soil but may have affected $\mathrm{Zn}$ and $\mathrm{Cu}$ uptake at the root surface or movement through the plant after uptake (Goh et al., 1997). Another possible explanation is that $\mathrm{P}$ application often reduces the colonization of roots by arbuscular mycorrhizal fungi (AMF) (Ova et al., 2015). In previous studies, $\mathrm{Zn}$ and $\mathrm{Cu}$ concentrations in shoots were reduced when AMF were absent (Kucey and Janzen, 1987). The current study also indicated 

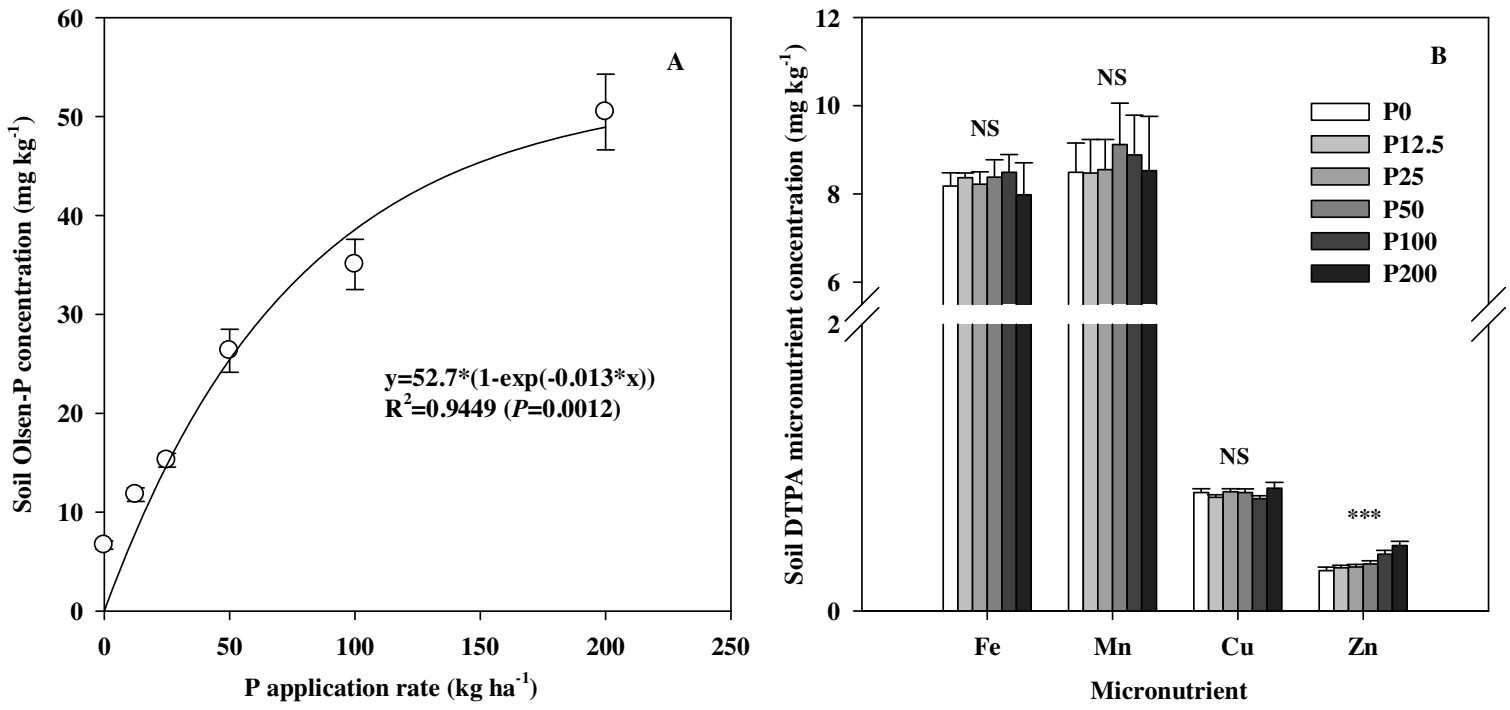

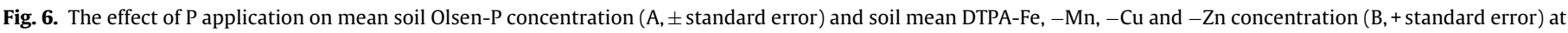
maturity. Values are the mean of three years. NS means not significant at $P>0.05$. ${ }^{* * *}$ indicates a significant difference among treatments at $P<0.0001$.

that the decrease in shoot $\mathrm{Zn}$ and $\mathrm{Cu}$ concentrations with increases in P application rate could not be explained by a "dilution effect" (in which concentrations decline as biomass increases), as suggested in a previous study (Zhu et al., 2002). This explanation was not supported because shoot biomass did not further increase but shoot Zn and $\mathrm{Cu}$ concentrations continued to decrease as the $\mathrm{P}$ application rate exceeded $12.5 \mathrm{~kg} \mathrm{P} \mathrm{ha}^{-1}$. As noted earlier, $\mathrm{P}$ application may have decreased $\mathrm{Zn}$ uptake by roots or $\mathrm{Zn}$ translocation from roots to shoots.

Shoot Fe and Mn concentrations were not significantly affected by $\mathrm{P}$ application rates at all growth stages. This agrees with previous reports concerning Fe in wheat (Zhang et al., 2012) and Mn in barley (Zhu et al., 2002). Although an increase in soil P availability slightly increased the Mn concentration in barley roots, Zhu et al. (2002) suggested that an increase in P supply may reduce Mn transportation from roots to shoots.

\subsection{Shoot micronutrient accumulation}

As the maize plants grew in the current study, the shoots accumulated increasing quantities of $\mathrm{P}, \mathrm{Fe}, \mathrm{Zn}, \mathrm{Cu}$, and $\mathrm{Mn}$ irrespective of $P$ application rate, but the amount accumulated (i.e., the content) at each growth stage differed depending on $P$ application rate. Within each growth stage, $P$ accumulation in shoots increased as the $\mathrm{P}$ application rate increased, but $\mathrm{Cu}$ and especially $\mathrm{Zn}$ accumulation in shoots decreased as the $\mathrm{P}$ application rate increased. The reduction in $\mathrm{Zn}$ accumulation in response to increases in $\mathrm{P}$ application differed from previous reports with wheat (Zhang et al., 2015). Zhang et al. (2015) found that $\mathrm{Zn}$ accumulation in wheat at the flowering and maturity stage increased as the $\mathrm{P}$ application rate increased from 0 to $50 \mathrm{~kg} \mathrm{P} \mathrm{ha}^{-1}$ and then decreased as the application rate increased further. In the current study, in contrast, $\mathrm{Zn}$ accumulation in maize shoots decreased as the $\mathrm{P}$ application rate increased from 0 to $200 \mathrm{~kg} \mathrm{ha}^{-1}$ during all growth stages. Cu accumulation in maize shoots was only slightly affected by $P$ application rates increased from 0 to $25 \mathrm{~kg} \mathrm{ha}^{-1}$ and then decreased at higher application rates. Differences between wheat and maize growing under similar environmental conditions may be ascribed to different nutrient requirements and different nutrient uptake efficiency. In this regard, maize is more tolerant to low $\mathrm{P}$ availability in soil than wheat (Postma and Lynch, 2011). This is ascribed at least in part to the stimulated root hair production (Ma et al., 2001) and mycorrhizal colonization of roots (Hagh et al., 2016) under P deficient conditions, which also enhance $\mathrm{Zn}$ and $\mathrm{Cu}$ uptake. Decreased mycorrhizal colonization with increased $P$ rates may result in an impaired Zn uptake by maize plants (Kothari et al., 1991). Our results indicate that the decline in $\mathrm{Zn}$ and $\mathrm{Cu}$ concentration is greater than the increase in shoot biomass with $\mathrm{P}$ application; thus it seems that there is an effect of $P$ fertilization on the concentration of these nutrients which is not ascribed to a dilution effect.

Shoot Fe and Mn accumulation steadily increased with P application rate from 0 to $200 \mathrm{~kg} \mathrm{P} \mathrm{ha}{ }^{-1}$, which is consistent with a previous study of cowpea crop (Nyoki and Ndakidemi, 2014). The increases of $\mathrm{Fe}$ and $\mathrm{Mn}$ accumulation in shoot were greatly ascribed to the increase in shoot biomass rather than nutrient concentration. The ratios of pre-anthesis to post-anthesis mineral content were not affected by $P$ application rate, which agrees with previous results in wheat (Zhang et al., 2015). In addition the competition between ions may also affect the uptake and accumulation of micronutrients. It was reported that ZRT-, IRT-like protein (ZIPs) and yellow stripe-like proteins (YSLs) are responsible for root $\mathrm{Zn}$ uptake and that these transport proteins can simultaneously transport Fe, Cu, Mn (Curie et al., 2009). The decrease of $\mathrm{Zn}$ accumulation may be caused by the increase of Fe and Mn accumulation which competed the same absorption site (Zhang et al., 2012).

\subsection{Micronutrient partitioning in maize tissues}

At maturity, the concentrations of $\mathrm{Cu}, \mathrm{Fe}$, and $\mathrm{Mn}$ in leaves were higher than in other shoot tissues, while $\mathrm{Zn}$ concentrations in grain and leaves were higher than in other shoot tissues. These results agree with previous studies (Bender et al., 2013). Marschner (2012) indicated that $\mathrm{Fe}, \mathrm{Mn}, \mathrm{Cu}$ and $\mathrm{Zn}$ in plant were moderate phloem mobility. However, compared with $\mathrm{Fe}, \mathrm{Mn}$, and $\mathrm{Cu}, \mathrm{Zn}$ remobilization from stem to grain is particularly critical for the accumulation of $\mathrm{Zn}$ in grain during reproductive growth in wheat (Kutman et al., 2012). For instance, it was reported that around $60-70 \%$ of the $\mathrm{Zn}$ content in grain was provided by $\mathrm{Zn}$ remobilization of pre-anthesis Zn uptake in wheat (Zhang et al., 2015). Zn and Cu concentrations in all tissues significantly decreased with increases in $\mathrm{P}$ application rate. $P$ application increased the concentration of $\mathrm{Fe}$ in stem, leaf, and rachis and increased the concentration of $\mathrm{Mn}$ in bract but had no significant effects on Fe and Mn concentrations in other plant parts. 
Irrespective of $\mathrm{P}$ rate, the average accumulation of $\mathrm{Cu}, \mathrm{Fe}$, and $\mathrm{Mn}$ in grain was 19,16 , and $13 \%$, respectively, of that in shoots, and the accumulation of $\mathrm{Zn}$ was from 58 to $71 \%$ of that in shoots. This partitioning was similar to that previously reported (Bender et al., 2013). The results further showed that the partitioning of $\mathrm{Zn}$ was affected by $\mathrm{P}$ application rate. Zinc harvest index, i.e. the ration of $\mathrm{Zn}$ content in grain to that in shoot, increased with increasing $P$ rates. This was consistent with a previous finding that more $\mathrm{Zn}$ was translocated to the grain and less was retained in the stover when maize was treated with increasing quantities of $\mathrm{P}$ (Verma and Minhas, 1987). Nicotianamine (NA) has been reported to be involved in the phloem loading and translocation of $\mathrm{Zn}$ (Deng et al., 2013), and an increase in NA stimulated $\mathrm{Zn}$ translocation and seed-loading (Lee et al., 2011). However, identifying the pathway by which $P$ application increases $\mathrm{Zn}$ translocation will require further research. The important fact is that $\mathrm{P}$ application promoted $\mathrm{Zn}$ translocation from stem, leaf, bract, and rachis to the grain of maize. Our results indicated that, even though P application decreased the $\mathrm{Zn}$ concentrations in grain and in all other shoot tissues of maize, it did not suppress $\mathrm{Zn}$ remobilization from vegetative tissues to grain, which agrees with results for wheat (Zhang et al., 2015).

\subsection{Bioavailability of micronutrients in grain}

The current study also showed that the average concentrations of $\mathrm{Zn}$ and $\mathrm{Fe}$ in grain were only $16.5 \mathrm{mg} \mathrm{kg}^{-1}$ and $17.4 \mathrm{mg} \mathrm{kg}^{-1}$, respectively. These values are far below the target values for biofortification ( $38 \mathrm{mg} \mathrm{kg}^{-1}$ for $\mathrm{Zn}$ and $60 \mathrm{mg} \mathrm{kg}^{-1}$ for $\mathrm{Fe}$ ) in maize (Bouis and Welch, 2010). The effects of $P$ application on the concentrations, contents, and partitioning of $\mathrm{P}, \mathrm{Zn}, \mathrm{Cu}, \mathrm{Fe}$, and $\mathrm{Mn}$ in maize will affect the bioavailability of these minerals in grain. Phytic acid (PA) is the primary P storage compound in seeds and PA accounts for up to $80 \%$ of the total P content in seed, while it impaired biological utilization by binding nutritionally important mineral such as Zn (Febles et al., 2002). Previous report had showed that the grain PA concentration was highly correlated with total $P$ concentration (Lolas et al., 1976). An increase in the $\mathrm{P}$ to $\mathrm{Zn}$ molar ratio indicated a decrease in the bioavailability of $\mathrm{Zn}$ in maize grain in response to $P$ application, which is consistent with previous reports on wheat (Zhang et al., 2012). The bioavailability of Fe in maize grain was also reduced by a high $\mathrm{P}$ supply. Because a reduction in $\mathrm{Zn}$ and $\mathrm{Fe}$ bioavailability in maize grain will likely have negative effects on human nutrition, research is needed to determine how to manage $P$ application so as to meet yield requirements while reducing the ratios of $\mathrm{P} / \mathrm{Zn}$ and $\mathrm{P} / \mathrm{Fe}$ in maize grain. To reduce the negative effects of $P$ application rates on the $Z n$ and Fe content in grain, growers should also consider applying $\mathrm{Zn}$ and Fe fertilizer in intensive maize production systems.

\section{Conclusion}

Phosphorus application significantly decreased shoot $\mathrm{Zn}$ and $\mathrm{Cu}$ concentrations but had no significant effects on shoot Fe and Mn concentrations at all growth stages. The ratios of pre-anthesis to post-anthesis nutrient accumulation were not affected by $P$ application rates except $\mathrm{Zn}$. P application had greater effects on the partitioning of $\mathrm{Zn}$ than on the partitioning of other nutrients and increased the partitioning of $\mathrm{Zn}$ to grain but decreased the partitioning of other nutrients to grain. This indicated that $Z n$ remobilization from vegetative tissues (stem, leaf, rachis, and bract) to grain was not suppressed by higher rates of $\mathrm{P}$ application. The increase in the ratios of $\mathrm{P} / \mathrm{Zn}$ and $\mathrm{P} / \mathrm{Fe}$ in grain as the $\mathrm{P}$ application rate increased indicated that increases in $\mathrm{P}$ application will reduce $\mathrm{Zn}$ and Fe bioavailability. These results indicate that $\mathrm{P}$ and micronutrients must be managed to meet both yield requirements and human nutritional requirements.

\section{Acknowledgements}

This research was funded by grants from the 973 Project (2015CB 150402), the National Science Foundation of China (31272252), the China Agriculture Research System (CARS-02), and the Innovative Group Grant of National Science Foundation of China (31121062). We thank Dr. Bruce Jaffee from USA for improving the English of the manuscript.

\section{References}

Bai, Z.H., Li, H.G., Yang, X.Y., Zhou, B.K., Shi, X.J., Wang, B.R., Li, D.C., Shen, J.B., Chen, Q., Qin, W., Oenema, O., Zhang, F.S., 2013. The critical soil P levels for crop yield, soil fertility and environmental safety in different soil types. Plant Soil 372, 27-37.

Banaj, D., Kovacevic, V., Simic, D., Seput, M., Stojic, B., 2006. Phosphorus impacts on yield and nutritional status of maize. Cereal Res. Commun. 34, 393-396.

Bender, R.R., Haegele, J.W., Ruffo, M.L., Below, F.E., 2013. Nutrient uptake, partitioning, and remobilization in modern: transgenic insect-protected maize hybrids. Agron. J. 105, 161-170.

Bouis, H.E., Welch, R.M., 2010. Biofortification-a sustainable agricultural strategy for reducing micronutrient malnutrition in the global south. Crop Sci. 50, 20-32.

Brown, W.L., Bressani, R., Glover, D.V., Hallauer, A.R., Johsnon, V.A., Qualset, C.O., Vietmeyer, N.D., 1988. Quality protein maize. In: Report of an Ad Hoc Panel of The Advisory Committee on Technology Innovation Board on Science and Technology for International Development National Research Council. National Academy Press, Washington DC.

Cakmak, I., 2002. Plant nutrition research: priorities to meet human needs for food in sustainable ways. Plant Soil 247, 3-24.

Colomb, B., Debaeke, P., Jouany, C., Nolot, J.M., 2007. Phosphorus management in low input stockless cropping systems: crop and soil responses to contrasting P regimes in a 36-year experiment in southern France. Eur. J. Agron. 26, 154-165.

Curie, C., Cassin, G., Couch, D., Divol, F., Higuchi, K., Le Jean, M., Misson, J., Schikora, A., Czernic, P., Mari, S., 2009. Metal movement within the plant: contribution of nicotianamine and yellow stripe 1-like transporters. Ann. Bot.-Lond. 103, 1-11.

Deng, F.L., Yamaji, N., Xia, J.X., Ma, J.F., 2013. A member of the heavy metal P-type ATPase OsHMA5 Is involved in xylem loading of copper in rice. Plant Physiol. $163,1353-1362$.

Febles, C.I., Arias, A., Hardisson, A., Rodrí́guez-Alvarez, C., Sierra, A., 2002. Phytic acid level in wheat flours. J. Cereal Sci. 36, 19-23.

Gibson, R.S., 2012. Zinc deficiency and human health: etiology, health consequences, and future solutions. Plant Soil 361, 291-299.

Goh, T.B., Banerjee, M.R., Tu, S.H., Burton, D.L., 1997. Vesicular arbuscular mycorrhizae-mediated uptake and translocation of $\mathrm{P}$ and $\mathrm{Zn}$ by wheat in a calcareous soil. Can. J. Plant Sci. 77, 339-346.

Guo, J.H., Liu, X.J., Zhang, Y., Shen, J.L., Han, W.X., Zhang, W.F., Christie, P., Goulding, K.W.T., Vitousek, P.M., Zhang, F.S., 2010. Significant acidification in major Chinese croplands. Science 327, 1008-1010.

Hagh, E.D., Mirshekari, B., Ardakani, M.R., Farahvash, F., Rejali, F., 2016. Optimizing phosphorus use in sustainable maize cropping via mycorrhizal inoculation. J. Plant Nutr. (Online), http://dx.doi.org/10.1080/01904167.2015.1086797.

Haldar, M., Mandal, L.N., 1981. Effect of phosphorus and zinc on the growth and phosphorus zinc, copper, iron and manganese nutrition of rice. Plant Soil 59, 415-425.

Hanway, J.J., 1962. Corn growth and composition in relation to soil fertility: II. Uptake of N, P, and $\mathrm{K}$ and their partition in different plant parts during the growing season. Agron. J. 54, 217-222.

Hegelund, J.N., Pedas, P., Husted, S., Schiller, M., Schjoerring, J.K., 2012. Zinc fluxes into developing barley grains: use of stable $\mathrm{Zn}$ isotopes to separate root uptake from remobilization in plants with contrasting Zn status. Plant Soil 361, $241-250$.

Izsáki, Z., 2014. Effect of phosphorus supplies on the nutritional status of maize (Zea mays L.). Commun. Soil Sci. Plan. 45, 516-529.

Karlen, D.L., Flannery, R.A., Sadler, E.J., 1988. Aerial accumulation and partitioning of nutrients by corn. Agron. J. 80, 232-242.

Kizilgoz, I., Sakin, E., 2010. The effects of increased phosphorus application on shoot dry matter, shoot P and Zn concentrations in wheat (Triticum durum L.) and maize (Zea mays L.) grown in a calcareous soil. Afr. J. Biotechnol. 9, 5893-5896.

Kothari, S.K., Marschner, H., Römheld, V., 1991. Contribution of the VA mycorrhizal hyphae in acquisition of phosphorus and zinc by maize grown in a calcareous soil. Plant Soil 131, 177-185.

Kovačević, V., Rastija, M., Šimić, B., Andrić, L., Kaučić, D., 2008. Phosphorus and potassium fertilization impacts on yield and nutritional status of maize. Cereal Res. Commun. 36, 43-46.

Kucey, R., Janzen, H.H., 1987. Effects of VAM and reduced nutrient availability on growth and phosphorus and micronutrient uptake of wheat and field beans under greenhouse conditions. Plant Soil 104, 71-78. 
Kutman, U., Kutman, B., Ceylan, Y., Ova, E., Cakmak, I., 2012. Contributions of root uptake and remobilization to grain zinc accumulation in wheat depending on post-anthesis zinc availability and nitrogen nutrition. Plant Soil 361, 177-187.

Le, C., Zha, Y., Li, Y., Sun, D., Lu, H., Yin, B., 2010. Eutrophication of lake waters in China: cost, causes, and control. Environ. Manage. 45, 662-668.

Lee, S., Persson, D.P., Hansen, T.H., Husted, S., Schjoerring, J.K., Kim, Y., Jeon, U.S., Kim, Y., Kakei, Y., Masuda, H., Nishizawa, N.K., An, G., 2011. Bio-available zinc in rice seeds is increased by activation tagging of nicotianamine synthase. Plant Biotechnol. J. 9, 865-873.

Lindsay, W.L., Norvell, W.A., 1978. Development of a DTPA soil test for zinc, iron, manganese, and copper. Soil Sci. Soc. Am. J. 42, 421-428.

Liu, Z., 1991. The Agricultural Chemistry and Micronutrients. Agricultural publisher of China, Beijing, pp. 93-232.

Lolas, G.M., Palamidis, N., Markakis, P., 1976. The phytic acid-total phosphorus relationship in barley oats, soybeans, and wheat. Cereal Chem. 53, 867-871.

Ma, Z., Bielenberg, D.G., Brown, K.M., Lynch, J.P., 2001. Regulation of root hair density by phosphorus availability in Arabidopsis thaliana. Plant Cell Environ. 24, 459-467.

Marschner, P., 2012. Marschner's Mineral Nutrition of Higher Plants, 3rd ed. Academic Press, pp. 77-78

Nuss, E.T., Tanumihardjo, S.A., 2010. Maize: a paramount staple crop in the context of global nutrition. Compr. Rev. Food Sci. Food Saf. 9, 417-436.

Nyoki, D., Ndakidemi, P.A., 2014. Influence of Bradyrhizobium japonicum and phosphorus on micronutrient uptake in cowpea. A case study of zinc (Zn) iron (Fe), copper (Cu) and manganese (Mn). Am. J. Plant Sci. 5, 427-435.

Ova, E.A., Kutman, U.B., Ozturk, L., Cakmak, I., 2015. High phosphorus supply reduced zinc concentration of wheat in native soil but not in autoclaved soil or nutrient solution. Plant Soil 393, 147-162.

Pearson, J.N., Rengel, Z., 1994. Distribution and remobilization of $\mathrm{Zn}$ and Mn during grain development in wheat. J. Exp. Bot. 45, 1829-1835.
Postma, J.A., Lynch, J.P., 2011. Theoretical evidence for the functional benefit of root cortical aerenchyma in soils with low phosphorus availability. Ann. Bot.-Lond. 107, 829-841.

Shukla, U.C., Singh, N., 1979. Phosphorus-copper relationship in wheat. Plant Soil 53, 399-402.

Stein, A.J., 2010. Global impacts of human mineral malnutrition. Plant Soil 335, $133-154$

Stewart, W.M., Dibb, D.W., Johnston, A.E., Smyth, T.J., 2005. The contribution of commercial fertilizer nutrients to food production. Agron. J. 97, 1-6.

Stomph, T.J., Jiang, W., Struik, P.C., 2009. Zinc biofortification of cereals: rice differs from wheat and barley. Trends Plant Sci. 14, 123-124.

Verma, T.S., Minhas, R.S., 1987. Zinc and phosphorus interaction in a wheat-maize cropping system. Fertil. Res. 13, 77-86.

Welch, R.M., 2002. The impact of mineral nutrients in food crops on global human health. Plant Soil 247, 83-90.

Xue, Y.F., Yue, S.C., Zhang, W., Liu, D.Y., Cui, Z.L., Chen, X.P., Ye, Y.L., Zou, C.Q., 2014. Zinc, iron, manganese and copper uptake requirement in response to nitrogen supply and the increased grain yield of summer maize. PLoS One 9, e93895.

Zhang, Y.Q., Deng, Y., Chen, R.Y., Cui, Z.L., Chen, X.P., Yost, R., Zhang, F.S., Zou, C.Q., 2012. The reduction in zinc concentration of wheat grain upon increased phosphorus-fertilization and its mitigation by foliar zinc application. Plant Soil 361, 143-152.

Zhang, W., Liu, D.Y., Li, C., Cui, Z.L., Chen, X.P., Russell, Y., Zou, C.Q., 2015. Zinc accumulation and remobilization in winter wheat as affected by phosphorus application. Field Crop. Res. 184, 155-161.

Zhu, Y.G., Smith, F.A., Smith, S.E., 2002. Phosphorus efficiencies and their effects on $\mathrm{Zn} \mathrm{Cu}$, and Mn nutrition of different barley (Hordeum vulgare) cultivars grown in sand culture. Aust. J. Agric. Res. 53, 211-216. 